\title{
Enhanced optical activity using the orbital angular momentum of structured light
}

\author{
Kayn A. Forbes $\mathbb{C}^{*}$ and David L. Andrews \\ School of Chemistry, University of East Anglia, Norwich Research Park, Norwich NR4 7TJ, United Kingdom
}

(Received 18 September 2019; published 6 November 2019)

\begin{abstract}
Recent molecular photonics studies have highlighted the significant role that phase-structured light possessing orbital angular momentum (OAM) can have when interacting with matter. These studies discovered chiroptical effects sensitive to both the magnitude and sign of the optical OAM in both the absorption and scattering of twisted photons by molecules and nanoparticles. Specifically, it has been shown how a structured beam engaging with electric-quadrupole transitions in the material allows a unique sensitivity to the helical-phase structure of twisted light. In this paper we highlight experimental methodologies and systems suitable to observe and quantify the chiroptical processes of Rayleigh and Raman optical activity, and the newly discovered circular-vortex differential scattering effect with structured light-including the importance of off-axis beam alignment, input beam intensity structure, multipolar moments, and scattering-angle dependencies. It is shown that with a judicious choice of experimental setup, chiroptical effects that scale with the topological charge $\ell$ or OAM of the input beam enable optical activity signals to be enhanced and significantly exceed those based solely on circularly polarized, unstructured light. The new technique thus offers a highly useful and important spectroscopic application of structured light. The more detailed role that perfect optical vortices with high OAM will play in such optical activity effects is now highlighted, to show where there is substantial scope for experimental application, specifically in vibrational optical activity and chiral spectroscopy.
\end{abstract}

DOI: 10.1103/PhysRevResearch.1.033080

\section{INTRODUCTION}

Optical activity is the intrinsic potential of chiral materials to exhibit discriminatory interactions with polarized light, according to their handedness [1,2]. For example, a righthanded molecule interacts differently with a right-handed circularly polarized beam than with a left-handed one. The most well-known examples include circular dichroism and optical rotation: the former signifies a differential absorption of left- and right-handed circularly polarized light, while the latter is a form of circular birefringence which (although it involves plane polarizations) is commonly interpreted in terms of differing refractive indices for superimposed left and right polarizations. The overriding principle of these chiroptical interactions is simple enough: both light and suitably structured matter may come in two different forms of handedness. Material handedness in this context stems from a chiral particle having no improper (rotation-reflection) axis of symmetry, while the helical structure that the electromagnetic field vectors trace out when propagating give circularly polarized light its own chirality (see Fig. 1). The interplay between these two forms of handedness in a light-matter interaction produces "natural" chiroptical effects [3]. It is important to make this distinction of natural chiroptical effects,

\footnotetext{
*Corresponding author: k.forbes@uea.ac.uk

Published by the American Physical Society under the terms of the Creative Commons Attribution 4.0 International license. Further distribution of this work must maintain attribution to the author(s) and the published article's title, journal citation, and DOI.
}

predominantly studied in the realm of chemical physics with molecular matter, from other areas such as metamaterials, quantum optics, and plasmonics [4-6], which seek to utilize achiral or exotic materials and optical systems in the pursuit of "mimicking" [7] chiral-optical phenomena. Of course, it has long been understood that achiral molecules can exhibit chiroptical behavior under suitable circumstances [8], such as in magnetic [9,10] and induced circular dichroism [11].

Circularly polarized photons convey a spin angular momentum (SAM) of $\pm \hbar$, where the \pm designates the direction that the electric field vector helically traces out in space [either clockwise (left-handed) or anticlockwise (right-handed)], and it is this helicity that gives circularly polarized light its handedness. In the early 1990s it was discovered that laser beams produced in the laboratory could also convey an additional optical orbital angular momentum (OAM) of $\pm|\ell| \hbar$, where $\ell$ is known as the topological charge or winding number, being able to take on any integer value [12]. These light beams propagate with a helical or twisted structure in their wave front, producing an optical vortex due to their azimuthal phase $e^{ \pm i \ell \phi}$. Therefore these structured beams also possess a handedness, twisting either to the right or to the left (see Fig. 1), irrespective of polarization. Studies on these optical vortices are flourishing, due to wide applicability including optical nanomanipulation, viscometry, free-space communications and information transfer, imaging, and microscopy; the reader is referred to a dedicated set of texts and articles on this large and ever-expanding field [13-18].

Studies of whether the handedness of an optical vortexthrough its OAM and topological charge $\pm|\ell|-$ could produce chiroptical interactions with chiral molecules in a similar manner to that of circularly polarized light were first 




FIG. 1. Different forms of handedness of light. Upper: Depictions of the electric (or magnetic) field vector in circularly polarized light. Lower: Wave front of twisted beams with their transverse phase distributions.

published nearly two decades ago [19] (though Padgett and Allen had already done experiments in 1993 with negative results that went unpublished, looking at this very question [20]). The published work proved that dipole transitions [engaging both electric-dipole (E1) and magnetic-dipole (M1) forms of coupling] could not produce discriminatory singlephoton absorption with twisted light. This conclusion was subsequently verified by experiments [21-23]. The theoretical studies were restrictive in that they did not allow for electric-quadrupole (E2) transition moments, which in their interaction with the radiation field are in general of a similar magnitude to the magnetic-dipole couplings. Despite the fact that E2 couplings deliver vanishing contributions to conventional circular dichroism and optical rotation in media with an isotropic distribution of local orientations, as in most fluids, their effect can become significant when coupled to structured light $[24,25]$. Recent studies [26,27] have identified that electric-quadrupole E2 interactions are in fact requisite in order to observe natural chiroptical interactions that depend on the handedness of a paraxial propagating optical vortex. Using quantum electrodynamical (QED) methodology, it was shown how the rate of single-photon absorption by a chiral molecule from a freely propagating and paraxial LaguerreGaussian beam (a specific type of structured OAM-carrying beam) can be discriminatory when the process takes place in anisotropic molecular systems. The effect, which engages interferences between electric dipole and quadrupole transitions, was termed circular-vortex dichroism (CVD) due to its similar and well-known relative circular dichroism (CD) [26]. Follow-up studies $[27,28]$ first revealed how discriminatory absorption can take place in achiral media, a supplementary analysis highlighting unique forms of Rayleigh and Raman optical activity using twisted photons.

Beyond these molecular QED studies looking at optical activity, a substantial amount of work on discriminatory interactions using the handedness of a twisted light beam has been carried out very recently. These studies, distinctly different from the "natural" QED ones highlighted above, have been able to induce chiroptical effects with OAM by utilizing the helicity-dependent intensity distributions that arise through the spin-orbit coupling of focused or scattered nonparaxial light with circular polarization [29]. Broadening the definition of "circular dichroism," fundamentally related effects have been identified in nonchiral nanostructures [30]; effects of a similar kind have also been discovered in achiral atomic matter [31] and chiral mesostructures [32], and they have been utilized in the characterization of material chirality [33] and spectroscopic probes of magnetism [34]. Other studies have investigated the exploitation of plasmonic coupling in material interactions with twisted light to engineer chiroptical effects [35-40], and recent theoretical work has predicted an x-ray analog to the CVD effect [41].

The two particularly important manifestations of optical activity that this paper is concerned with are the chiroptical processes of Rayleigh and Raman optical activity [42]: the differential scattering of circularly polarized light by chiral molecules. Raman optical activity (confusingly given the acronym "ROA" in the literature) [43] is almost exclusively involved with vibrational transitions. Alongside vibrational circular dichroism (VCD) in the infrared, ROA represents the key methodology for studying the structure of chiral molecules through vibrational optical activity (VOA). The virtues of ROA include proportionately stronger signals than VCD; the opportunity to observe, using visible wavelengths, structural features of chirality directly associated with the nuclear framework of chiral species; also the possibility of using resonance enhancement, tuning toward the absorption bands of specific functional groups in order to deduce the chirality of their immediate intramolecular environment. Exhaustive reviews of the application of ROA and VOA can be found elsewhere [44-53], but it is worth noting in particular their extremely proficient application in determining the molecular structures and motions of important biomolecules, such as viruses, carbohydrates, and proteins, even as large as insulin [54]. Optical activity effects and the signals produced in experiments are inherently much weaker than the usually 
dominant electric-dipole couplings due to the fact they stem from interferences between electric-dipole and the higherorder electric-quadrupole and magnetic-dipole interactions with the field. As such, a great deal of research effort has gone into enhancing optical activity effects and signals in spectroscopic applications [55-61].

The aim of this paper is to significantly build on the recently discovered circular-vortex differential scattering (CVDS) Rayleigh and Raman optical activity effect [28] by highlighting how, using high-OAM beams, the ensuing optical activity and rate of differential scattering (both Raman and Rayleigh) can be significantly enhanced in comparison to the currently utilized zero-OAM unstructured light. Further insights are provided by studying the distinct scattering-angle dependence of CVDS, particularly in comparison to standard circular-differential scattering, and which in consequence lead to insights in potential experimental methodologies to observe and quantify the effect. Moreover, we explicitly develop the underlying theory to elicit the conditions under which these interactions may be observed in general, and highlight the important role that perfect optical vortices and beams with large values of optical OAM will play in spectroscopic applications of structured light.

In Sec. II we introduce the circular-vortex differential scattering (CVDS) effect in a general form, and highlight its applicability to both Rayleigh and Raman optical activity; Sec. III looks at the dependence of CVDS on the input beam OAM and the scattering angle of the output light, specifically highlighting the different angle dependencies of structured and nonstructured light; Sec. IV highlights and discusses the intricacies of experimental observation of CVDS; we end with a discussion and summary in Sec. V.

\section{RAYLEIGH AND RAMAN OPTICAL ACTIVITY WITH STRUCTURED LIGHT}

\section{A. General formalism}

In standard Rayleigh and Raman optical activity the scattering rate of circularly polarized light (and hence the intensity of scattered light) depends on the relative helicity of the incident light and the handedness of the optically active molecule: a right-handed molecule will scatter right-handed circularly polarized light at a different rate from left-handed, for example. The mechanism for this circular-differential scattering (CDS) stems from the interferences of the wellknown frequency-dependent electric-dipole molecular polarizability tensor $\alpha_{i j}\left(\omega,-\omega^{\prime}\right)$ with both the mixed electricdipole magnetic-dipole gyration $G_{i j}\left(\omega,-\omega^{\prime}\right)$ and electricdipole electric-quadrupole polarizability $A_{i j k}\left(\omega,-\omega^{\prime}\right)$ tensors, i.e., " $\alpha G$ " and " $\alpha A$ " coupling [1,2] (the input optical frequency denoted by $\omega$ and the scattered light frequency $\left.\omega^{\prime}\right)$. For all molecules, $\alpha_{i j}\left(\omega,-\omega^{\prime}\right)$ has nonvanishing tensor components, whereas only chiral molecules can support the corresponding interferences between $G_{i j}\left(\omega,-\omega^{\prime}\right)$ and $A_{i j k}\left(\omega,-\omega^{\prime}\right)$ polarizabilities with $\alpha_{i j}\left(\omega,-\omega^{\prime}\right)$. These polarizabilities take on the following forms, where $\boldsymbol{\mu}^{m 0}$ and $\boldsymbol{m}^{m 0}$ are the transition electric- and magnetic-dipole moments, respectively, between the initial $|0\rangle$ and final $|m\rangle$ material states-and it is important to note that only $A_{i j k}\left(\omega,-\omega^{\prime}\right)$ involves electric-quadrupole transition moments $Q_{j k}^{m 0}$ :

$$
\begin{aligned}
& \alpha_{i j}^{m 0}\left(\omega,-\omega^{\prime}\right)=\sum_{r}\left[\frac{\mu_{i}^{m r} \mu_{j}^{r 0}}{E_{r 0}-\hbar \omega}+\frac{\mu_{j}^{m r} \mu_{i}^{r 0}}{E_{r 0}+\hbar \omega^{\prime}}\right], \\
& G_{i j}^{m 0}\left(\omega,-\omega^{\prime}\right)=\sum_{r}\left[\frac{\mu_{i}^{m r} m_{j}^{r 0}}{E_{r 0}-\hbar \omega}+\frac{m_{j}^{m r} \mu_{i}^{r 0}}{E_{r 0}+\hbar \omega^{\prime}}\right], \\
& A_{i j k}^{m 0}\left(\omega,-\omega^{\prime}\right)=\sum_{r}\left[\frac{\mu_{i}^{m r} Q_{j k}^{r 0}}{E_{r 0}-\hbar \omega}+\frac{Q_{j k}^{m r} \mu_{i}^{r 0}}{E_{r 0}+\hbar \omega^{\prime}}\right] .
\end{aligned}
$$

As is evident on inspection, these three tensors involve coupling of E1 ${ }^{2}$, E1M1, and E1E2 form, respectively. The superscripts in the polarizabilities (2.1)-(2.3) denote transitions occurring from the ground state $|0\rangle$ to the final state $|m\rangle$, through a virtual intermediate state $|r\rangle ; E_{r 0}=E_{r}-E_{0}$ represents the difference between the intermediate energy state and the initial energy state of the material; the Latin subscript indices denote standard suffix notation for vector components, where summation over repeated indices is assumed implicit in rate expressions throughout the paper.

The rate of differential Rayleigh and Raman scattering of OAM-conveying Laguerre-Gaussian light [28] has recently been derived using the multipolar Power-Zienau-Woolley Hamiltonian formalism [2,62]. In this work it was found that the well-known CDS for both Rayleigh and Raman optical activity is supplemented by a further chiroptical effect, if the incident light possesses OAM. This mechanism has been termed circular-vortex differential scattering (CVDS) as it depends on material chirality and the sign of $\ell$ (as well as the circular polarization), and importantly it only contributes through $\alpha A$ coupling.

Consider an initial system state consisting of a molecule $\left|E_{0}(\xi)\right\rangle$, and optical mode occupancy $|n(k, \eta, \ell, p)\rangle$ of $n$ LG photons in the mode $(k, \eta, \ell, p)$, where $k$ is the magnitude of the optical wave vector, $\eta$ is the beam polarization, $\ell$ the winding number (topological charge), and $p$ a radial distribution index, the secondary index of the Laguerre polynomial of order $\ell$. In the course of the fundamental CVDS interaction, the system progresses to a final state $|\{n-1\}(k, \eta, \ell, p)\rangle\left|1\left(\boldsymbol{k}^{\prime}, \eta^{\prime}\right)\right\rangle\left|E_{m}(\xi)\right\rangle$, where the scattered photon is in some mode $\left(\boldsymbol{k}^{\prime}, \eta^{\prime}\right)$. The full derivation for the scattered radiant intensity for CVDS in isotropic systems (such as a liquid) is given in the Appendix. After much manipulation, the intensity elements that arise specifically through $\alpha A$ coupling and the beam structure can be recast in the following form:

$$
\begin{aligned}
I^{\alpha A}\left(\boldsymbol{k}^{\prime}\right)= & \beta i k\left\{\left(\hat{\boldsymbol{k}}^{\prime} \cdot \boldsymbol{c}\right)+\left(\hat{z} \cdot \overline{\boldsymbol{e}}^{\prime}\right)\left(\boldsymbol{c} \cdot \boldsymbol{e}^{\prime}\right)+\left(\overline{\boldsymbol{b}^{\prime}} \cdot \overline{\boldsymbol{e}}\right)\left(\boldsymbol{e} \cdot \boldsymbol{e}^{\prime}\right)\right. \\
& -\left(\boldsymbol{b} \cdot \boldsymbol{e}^{\prime}\right)\left(\overline{\boldsymbol{e}} \cdot \overline{\boldsymbol{e}^{\prime}}\right)-i k^{-1}\left[f_{|\ell|, p}^{-1}(r) \partial_{r} f_{|\ell|, p}(r)-r^{-1}\right] \\
& \times\left[\left(\hat{\boldsymbol{r}} \cdot \overline{\boldsymbol{e}}^{\prime}\right)\left(\boldsymbol{c} \cdot \boldsymbol{e}^{\prime}\right)-\left(\hat{\boldsymbol{r}} \times \boldsymbol{e} \cdot \boldsymbol{e}^{\prime}\right)\left(\overline{\boldsymbol{e}} \cdot \overline{\boldsymbol{e}}^{\prime}\right)\right] \\
& +\ell k^{-1} r^{-1}\left(\hat{\boldsymbol{\phi}} \cdot \overline{\boldsymbol{e}}^{\prime}\right)\left(\boldsymbol{c} \cdot \boldsymbol{e}^{\prime}\right) \\
& \left.-\ell k^{-1} r^{-1}\left(\hat{\boldsymbol{\phi}} \times \boldsymbol{e} \cdot \boldsymbol{e}^{\prime}\right)\left(\overline{\boldsymbol{e}} \cdot \overline{\boldsymbol{e}^{\prime}}\right)\right\} \alpha_{\lambda \mu}^{m 0} A_{\lambda \mu}^{\prime m 0},
\end{aligned}
$$

where $\beta=I_{0}(r) k^{4} / 240 \varepsilon_{0}^{2} \pi^{2}$ and $I_{0}(r)=n \hbar c^{2} k f_{|\ell|, p}^{2}(r) / V$ is the input irradiance; $\boldsymbol{e}^{\prime}$ and $\boldsymbol{e}$ are unit electric polarization vectors for the scattered (indicated by a prime) and incident 
photon, respectively ( $\boldsymbol{b}$ relates to the magnetic field), and $\boldsymbol{c}=\boldsymbol{e} \times \overline{\boldsymbol{e}}$ (where the overbar denotes the complex conjugate); $\alpha_{\lambda \mu}^{m 0}$ is the orientationally averaged [63] form of the molecular polarizability (2.1), and similarly the averaged form of (2.3) is $A_{\lambda \mu}^{\prime m 0}=\varepsilon_{\lambda \alpha \beta} A_{\alpha \beta \mu}^{m 0} ; \hat{\boldsymbol{\phi}}$ and $\hat{\boldsymbol{r}}$ are the azimuthal and radial unit vectors, respectively, with $r$ the radial distance of the scattering molecule from the beam axis. In the definition of the input irradiance, $V$ is an arbitrary quantization volume and $f_{|\ell|, p}(r)$ is a radial distribution function.

The terms dependent on $\hat{\boldsymbol{r}}$ will be present for any nonplane-wave laser beam, simply signaling a dependence on radial intensity gradients. In this case it is expedient to note that they are either nondiscriminatory with regard to the circularpolarization state (i.e., they do not engage in optical activity) or unobservable imaginary quantities (see Appendix). Furthermore, they are also independent of $\ell$-although it is worth pointing out that the term in $\hat{\boldsymbol{r}} / r$ does originate from the helical phase, and would therefore be absent in non-OAM-carrying beams. In light of this we may neglect the $\hat{\boldsymbol{r}}$-dependent terms from now on.

The corresponding scattered intensity contribution from $\alpha G$ coupling for structured light is similarly

$$
\begin{aligned}
I^{\alpha G}\left(\boldsymbol{k}^{\prime}\right)= & -\frac{\beta}{c}\left[\left\{4\left(\overline{\boldsymbol{e}}^{\prime} \cdot \boldsymbol{e}\right)\left(\boldsymbol{e}^{\prime} \cdot \overline{\boldsymbol{b}}\right)+4\left(\boldsymbol{e}^{\prime} \cdot \overline{\boldsymbol{e}}\right)\left(\overline{\boldsymbol{b}}^{\prime} \cdot \boldsymbol{e}\right)-(\boldsymbol{e} \cdot \overline{\boldsymbol{b}})\right.\right. \\
& \left.-\left(\boldsymbol{e}^{\prime} \cdot \overline{\boldsymbol{b}}^{\prime}\right)-\left(\overline{\boldsymbol{e}}^{\prime} \cdot \overline{\boldsymbol{b}}\right)\left(\boldsymbol{e} \cdot \boldsymbol{e}^{\prime}\right)-\left(\overline{\boldsymbol{e}} \cdot \overline{\boldsymbol{b}}^{\prime}\right)\left(\boldsymbol{e} \cdot \boldsymbol{e}^{\prime}\right)\right\} \alpha_{\lambda \lambda}^{m 0} G_{\mu \mu}^{m 0} \\
& +\left\{-2\left(\overline{\boldsymbol{e}}^{\prime} \cdot \boldsymbol{e}\right)\left(\boldsymbol{e}^{\prime} \cdot \overline{\boldsymbol{b}}\right)-2\left(\boldsymbol{e}^{\prime} \cdot \overline{\boldsymbol{e}}\right)\left(\overline{\boldsymbol{b}}^{\prime} \cdot \boldsymbol{e}\right)\right. \\
& +3(\boldsymbol{e} \cdot \overline{\boldsymbol{b}})+3\left(\boldsymbol{e}^{\prime} \cdot \overline{\boldsymbol{b}}^{\prime}\right)+3\left(\overline{\boldsymbol{e}}^{\prime} \cdot \overline{\boldsymbol{b}}\right)\left(\boldsymbol{e} \cdot \boldsymbol{e}^{\prime}\right) \\
& \left.\left.+3\left(\overline{\boldsymbol{e}} \cdot \overline{\boldsymbol{b}}^{\prime}\right)\left(\boldsymbol{e} \cdot \boldsymbol{e}^{\prime}\right)\right\} \alpha_{\lambda \mu}^{m 0} G_{\lambda \mu}^{m 0}\right],
\end{aligned}
$$

where $G_{\lambda \mu}^{m 0}$ is the averaged version of the electric-magnetic dipole mixed polarizability tensor (2.2). Here we reiterate that as both $\alpha_{\lambda \mu}^{m 0}$ and $G_{\lambda \mu}^{m 0}$ only involve dipole interactions (namely E1 and M1, lacking any E2 interactions), (2.5) does not involve any dependence on the OAM of the beam through an $\ell$ dependence, unlike the contribution from $\alpha A$ (2.4). It is to be understood that the total optical activity for Rayleigh and Raman optical activity is a sum of the two distinct contributions (2.4) and (2.5).

In comparing the form of the result previously given for CVDS in [28], the new result (2.4) is of a general form and therefore more amenable to manipulation in order to extract the physics of CVDS. If the beam were to possess no OAM, such that $\ell=0$, our results (2.4) and (2.5) would reduce to the well-known results of Rayleigh and Raman optical activity for a Gaussian beam. The new result (2.4) also includes the terms dependent on the radial gradient of the beam, which as shown has no direct connection to the optical OAM nor possesses optical activity behavior.

Experiments are usually concerned with the particular cases where $\boldsymbol{e}^{\prime}$, the polarization of the scattered photon, is resolved for components either in the $\hat{\boldsymbol{k}} \hat{\boldsymbol{k}}^{\prime}$ plane $\boldsymbol{e}^{\prime}{ }_{(\|)}$or normal $\boldsymbol{e}^{\prime}(\perp)$ to it (Fig. 2).

The scattered intensities (2.4) and (2.5) can then be taken one step further by introducing the dimensionless circular intensity difference (CID) first proposed by Barron and

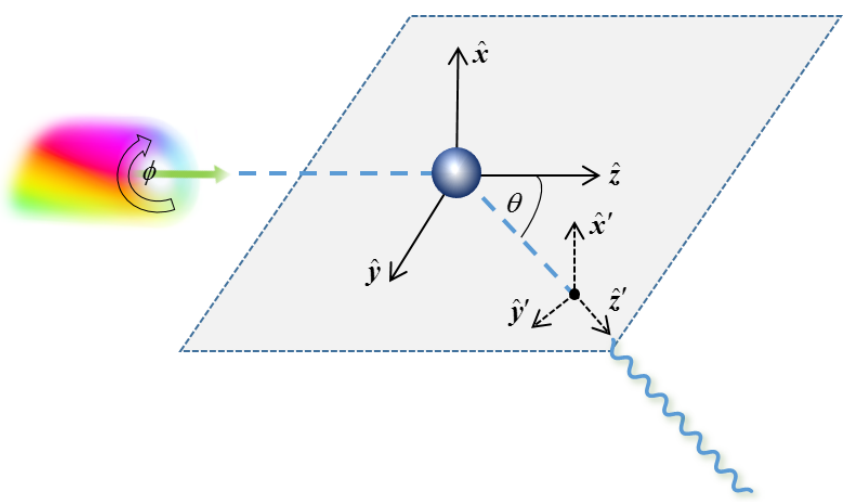

FIG. 2. Schematic scattering geometry for the scattering of an input twisted LG mode photon, by a molecule or nanoparticle, at an arbitrary angle $\theta$. For the input light $\hat{\boldsymbol{k}}=\hat{\boldsymbol{z}}$; for the scattered light $\hat{\boldsymbol{k}}^{\prime}=\hat{z}^{\prime}$.

Buckingham,

$$
\Delta_{\mu}(\theta)=\frac{I_{\theta}(\mathrm{R} \rightarrow \mu)-I_{\theta}(\mathrm{L} \rightarrow \mu)}{I_{\theta}(\mathrm{R} \rightarrow \mu)+I_{\theta}(\mathrm{L} \rightarrow \mu)}(\mu=\perp, \|),
$$

where $\mathrm{R}$ and $\mathrm{L}$ represent the right-handed and left-handed circular-polarization state of the incident light, respectively, and $\perp$ corresponds to scattered light that is polarized transverse to the scattering $\hat{\boldsymbol{k}} \hat{\boldsymbol{k}}^{\prime}$ plane, while $\|$ corresponds to scattered light polarized in the $\hat{\boldsymbol{k}} \hat{\boldsymbol{k}}^{\prime}$ plane. The quantity in the numerator of (2.6) simply represents the polarizationdependent differences in the circular differential scattering contributions (2.4) and (2.5), because the scattering rate of circularly polarized photons for standard, non-optically-active $\alpha^{2}$ scattering is the same for left- and right-handed polarizations; the quantity in the denominator is, by the same logic, dominated by the standard Rayleigh and Raman scattering effect for nonresonant light:

$$
\begin{aligned}
I^{\alpha^{2}}\left(\boldsymbol{k}^{\prime}\right)= & \beta\left[\left\{4\left(\boldsymbol{e} \cdot \overline{\boldsymbol{e}}^{\prime}\right)\left(\overline{\boldsymbol{e}} \cdot \boldsymbol{e}^{\prime}\right)-\left(\boldsymbol{e} \cdot \boldsymbol{e}^{\prime}\right)\left(\overline{\boldsymbol{e}} \cdot \overline{\boldsymbol{e}^{\prime}}\right)-1\right\} \alpha_{\lambda \lambda}^{m 0} \alpha_{\mu \mu}^{m 0}\right. \\
& \left.+\left\{3-2\left(\boldsymbol{e} \cdot \overline{\boldsymbol{e}}^{\prime}\right)\left(\overline{\boldsymbol{e}} \cdot \boldsymbol{e}^{\prime}\right)+3\left(\boldsymbol{e} \cdot \boldsymbol{e}^{\prime}\right)\left(\overline{\boldsymbol{e}} \cdot \overline{\boldsymbol{e}^{\prime}}\right)\right\} \alpha_{\lambda \mu}^{m 0} \alpha_{\lambda \mu}^{m 0}\right] .
\end{aligned}
$$

It has previously been discovered how both Raman and Rayleigh optical activity have an important sensitivity to the scattering angle $\theta$ [64]. By studying this angle dependence, it has been shown how it is possible to experimentally verify the different contributions to circular-differential scattering (the scattering of nonvortex light) associated with the E2 and M1 couplings (i.e., the $A$ and $G$ tensors, respectively) and their relative contributions. It is evident that a similar analysis for the CVDS effect will be of special interest due to the distinct role that E2 interactions play for twisted light. By evaluating the polarization scalar products in the above intensity expressions we derive the CVDS effect for both scattering scenarios and for an arbitrary scattering angle $\theta$ (often in scattering theories the angle is restricted to either forward $180^{\circ}$, backward $0^{\circ}$, or at $90^{\circ}$ [1]).

Using rigorous quantum electrodynamical methods (detailed in the Appendix) we thus derive a set of parameters from (2.4), (2.5), and (2.7) that are expressed in terms of 
the molecular response tensors, $\alpha_{\lambda \mu}, G_{\lambda \mu}^{\prime}=i G_{\lambda \mu}$, and $A_{\lambda \mu}{ }^{\prime}=$ $\varepsilon_{\lambda \alpha \beta} A_{\alpha \beta \mu}$ :

$$
\begin{gathered}
a=\frac{4}{c}\left(-\omega \alpha_{\lambda \mu} A^{\prime}{ }_{\lambda \mu}-\alpha_{\lambda \lambda} G_{\mu \mu}^{\prime}+3 \alpha_{\lambda \mu} G_{\lambda \mu}^{\prime}\right) \\
b=\frac{2}{c}\left(-3 \omega \alpha_{\lambda \mu} A^{\prime}{ }_{\lambda \mu}+5 \alpha_{\lambda \lambda} G_{\mu \mu}^{\prime}-5 \alpha_{\lambda \mu} G_{\lambda \mu}^{\prime}\right), \\
c=\frac{2}{c}\left(3 \omega \alpha_{\lambda \mu} A_{\lambda \mu}^{\prime}+3 \alpha_{\lambda \lambda} G_{\mu \mu}^{\prime}+\alpha_{\lambda \mu} G_{\lambda \mu}^{\prime}\right) \\
d=\frac{\ell}{r} \alpha_{\lambda \mu} A_{\lambda \mu}^{\prime} \cos \phi \\
f=6 \alpha_{\lambda \mu} \alpha_{\lambda \mu}-2 \alpha_{\lambda \lambda} \alpha_{\mu \mu} \\
g=\alpha_{\lambda \mu} \alpha_{\lambda \mu}+3 \alpha_{\lambda \lambda} \alpha_{\mu \mu} .
\end{gathered}
$$

In deriving these parameters we have made use of the following relations: $\boldsymbol{c}^{\mathrm{L} / \mathrm{R}}=\mp i \hat{\boldsymbol{k}}, \boldsymbol{e}^{\mathrm{L} / \mathrm{R}}=\sqrt{2}^{-1}\left(\boldsymbol{e}_{(\|)} \pm i \boldsymbol{e}_{(\perp)}\right)$, $\boldsymbol{b}^{(\mathrm{L} / \mathrm{R})}=\mp i \boldsymbol{e}^{(\mathrm{L} / \mathrm{R})}$, and the well-known coordinate transformation for cylindrical to Cartesian unit vectors. The CID rate (2.6) that includes the distinct CVDS contribution (2.11) can be secured for radiation with polarization components in the $\hat{\boldsymbol{k}} \hat{\boldsymbol{k}}^{\prime}$ plane in terms of (2.8)-(2.13) and succinctly expressed as

$$
\Delta_{\|}(\ell, \theta)=\frac{a+b \cos \theta+c \cos ^{2} \theta+d \sin 2 \theta}{f+g \cos ^{2} \theta},
$$

while the corresponding form for scattered light whose polarization component is completely transverse to the $\hat{\boldsymbol{k}} \hat{\boldsymbol{k}}^{\prime}$ plane is

$$
\Delta_{\perp}(\theta)=\frac{a+b \cos \theta+c}{f+g} .
$$

From the two results (2.14) and (2.15), before any analysis of the angle dependence, we immediately discover that the CVDS effect (2.11) can only be observed for scattered light with polarization components in the $\hat{\boldsymbol{k}} \hat{\boldsymbol{k}}^{\prime}$ plane: regardless of the scattering angle, if the scattered light is analyzed for a component polarized in the transverse plane there is no CVDS effect. This follows from the fact that CVDS depends on the topological charge and hence the orbital angular momentum of the input beam, which has no components in the transverse plane. The result therefore offers an extremely simple way to experimentally verify and quantify the CVDS effect. With a polarization analyzer positioned so as to measure transverse components, the CID result will not change upon changing the incident light's OAM; with a polarization analyzer set up to measure the $\hat{\boldsymbol{k}} \hat{\boldsymbol{k}}^{\prime}$-plane components, the CID will change upon changing the OAM of the input beam, including departures from the case of when $\ell=0$, where the standard CDS effect will be observed. In conducting such experiments, it will of course be necessary to take into account variations in the radial intensity distributions of the input, according to the forms of the associated Laguerre polynomials-for which exact analytical expressions are well known.

\section{B. Vibrational dependence}

Up until this point, the given results apply specifically to Rayleigh (elastic) scattering. For Raman scattering, the same CID expressions apply, but with molecular property tensors replaced by vibrational Raman transition tensors between vibrational states. These changes are well known and established in the literature [1,2], but it is worth outlining them here, particularly as the Raman form of optical activity is much more important than the Rayleigh form.

For chiral molecules, each allowed Raman transition will generate nonzero components of transition polarizabilities of not only E1 ${ }^{2}$ form but also E1M1 and E1E2. Each corresponding Raman polarizability tensor describes transitions between an initial vibrational state $|\nu\rangle$ and final vibrational state $\left|v^{\prime}\right\rangle$; so, for example, $\alpha_{\lambda \mu}^{m 0}$ is replaced by $\left\langle v^{\prime}\left|\alpha_{i j}(Q)\right| v\right\rangle$, where $\alpha_{i j}(Q)$ is the electronic polarizability whose components depend parametrically on the normal vibrational coordinates $Q$. This effective polarizability $\alpha_{i j}(Q)$ may be expanded in a Taylor series about the equilibrium position $Q_{\mathrm{e}}$, where the leading term is

$$
\alpha_{\lambda \mu}^{m 0}\left(\omega,-\omega^{\prime}\right)=\left.\frac{\partial \alpha_{\lambda \mu}(Q)}{\partial Q}\right|_{Q_{\mathrm{e}}}\left\langle\nu^{\prime}\left|Q-Q_{\mathrm{e}}\right| v\right\rangle .
$$

The Raman intensity is therefore determined by the variation of the polarizability tensor with a normal coordinate of vibration; an analogous treatment leads to similar forms of $G_{\lambda \mu}^{m 0}=\left\langle v^{\prime}\left|G_{\lambda \mu}(Q)\right| v\right\rangle$ and $A_{\lambda \mu}^{\prime m 0}=\left\langle v^{\prime}\left|A_{\lambda \mu}(Q)\right| v\right\rangle$. When the appropriate substitutions are made in Eqs. (2.14) and (2.15), the vibrational part $\left\langle v^{\prime}\left|Q-Q_{\mathrm{e}}\right| v\right\rangle$ in each numerator cancels with the same factor in the denominator, so that the given equations can legitimately be applied with the coefficients $a-f$ directly reinterpreted in terms of the transition tensors $\alpha_{\lambda \mu}^{m 0}, G_{\lambda \mu}^{m 0}$, and $A_{\lambda \mu}^{\prime m 0}$. All the ensuing results in this article are therefore readily applicable to both Rayleigh and Raman forms of optical activity by following these simple modifications.

On application to the results of ROA spectroscopy, circular difference spectra can be recorded and the variation with scattering angle determined for each vibrational band. Here, distinctive differences will arise in the degrees of sensitivity of the results, through their dependence on $A_{\lambda \mu}^{\prime m 0}$, to registration of the phase structure of the structured light. With increasing topological charge $\ell$, each active vibration will respond differently to the tighter phase gradient through its engagement with their E2 transition moments. For example, in a chiral polysubstituted benzene, it may be anticipated that the asymmetric in-plane deformation mode of the aromatic center will be more sensitive, and so give a larger CVDS signal, than vibrations of the peripheral groups. Worth mentioning here is a theoretical study that highlighted how, because of the optical OAM, new selection rules for standard Raman scattering with vortex light lead to activation of silent phonon modes in cubic crystals [65].

\section{SCATTERING-ANGLE AND OAM DEPENDENCE}

The CIDs (2.14) and (2.15) have both been derived in a form that allows for an arbitrary scattering-angle $\theta$ analysis, and include all contributions to the optical activity scattering from both $\alpha G$ and $\alpha A$ coupling normalized against the dominate $\alpha^{2}$ scattering. In this analysis we are primarily concerned with the role of structured light in optical activity and specifically the CVDS effect. We now restrict consideration to the optical activity effects solely due to $\alpha A$ interactions (2.4), 
discarding the $\alpha G$ and $\alpha^{2}$ contributions, as neither of these engage the helical-phase structure and OAM of the structured beam. Although these would be important for an empirical analysis, we are concerned with extracting the novel physics of this new form of optical activity that has been discovered for structured light.

Using (2.14) and (2.15), along with the parameters (2.8)(2.15), we produce the following differences in scattered intensity:

$$
\begin{aligned}
\Delta I^{\alpha A}\left(\boldsymbol{k}^{\prime}, \|\right)= & \beta k\left[3 \cos ^{2} \theta-3 \cos \theta-2+\frac{\ell}{2 k r} \sin 2 \theta \cos \phi\right] \\
& \times \alpha_{\lambda \mu}^{m 0} A_{\lambda \mu}^{\prime m 0}
\end{aligned}
$$

and

$$
\Delta I^{\alpha A}\left(\boldsymbol{k}^{\prime}, \perp\right)=\beta k[1-3 \cos \theta] \alpha_{\lambda \mu}^{m 0} A_{\lambda \mu}^{\prime m 0} .
$$

As already highlighted in the previous section, scattered light with a purely transverse polarization with respect to $\boldsymbol{k}^{\prime}$ (3.2) exhibits no CVDS effect, and therefore we concentrate now on (3.1).

In (3.1), the final term in square brackets represents the CVDS term, and for beams without OAM $\ell=0$ it vanishes; the other three terms correspond to the standard and wellknown CDS effect, and the total scattered intensity difference is a sum of both CDS and CVDS. There are five distinct and important differences that are worth highlighting between the CVDS effect and standard CDS that exist in the former, arising from the OAM dependence:

(a) The CVDS effect has a different scattering-angle dependence from the CDS term. The CVDS effect is at a maximum when $\theta=\frac{\pi}{4}, \frac{3 \pi}{4}$, while the maximum for CDS occurs at $\theta=\pi$ (and at $\theta=\pi$ the CVDS effect is zero).

(b) CVDS importantly depends linearly on $\ell$, and so a beam with a vortex charge of unity and that twists to the left $\ell=+1$ gives the opposite result for the same vortex that twists to the right $\ell=-1$. This is therefore a chiroptical effect that is dependent on the sign of the optical OAM.

(c) Further to point (b) and crucially, CVDS also depends on the magnitude of $\ell$. As $\ell$ increases in magnitude the correspondingly more heavily weighted contribution of CVDS will begin to dominate the standard CDS contribution to the scattered intensity difference. It is important to recall that $\ell$ is an unbounded integer, with experimentally realized vortex beams having already achieved values over 5000 [66], and so the mechanism of CVDS represents a way to significantly enhance the optical activity signals in experiment.

(d) In comparison to standard CDS, CVDS is also dependent on the factor of $(\mathrm{kr})^{-1}$, that is, the inverse of the product of the wave number of the incident light and the radial position of chiral particle in the transverse profile of the beam. As a result of the Laguerre polynomial structure imposed on a Gaussian profile, an LG beam typically comprises a set of concentric rings of intensity, centered on the beam axis. Often, attention focuses on beams with $p=0$, where for $\ell \geqslant 1 \mathrm{a}$ single "donut" beam forms. In such a case the general result is easy to see: particles positioned closer to the inside ring of the intensity donut (where $r$ is small and the gradient of the fields



FIG. 3. Plot of differential scattered intensity of light with $\hat{\boldsymbol{k}} \hat{\boldsymbol{k}}^{\prime}$ plane polarization components against scattering angle (in degrees). Solid line (purple) represents the combined CDS and CVDS effect for an $\ell=1$ incident beam and $k r=1$; the dashed curve (green) represents the CVDS effect alone for $\ell=1$; and the dotted curve (red) is solely the CDS contribution (i.e., $\ell=0$ ).

is larger) will produce a CVDS effect that can contribute more significantly than the CDS term.

(e) Finally, the CVDS term is dependent on the cosine of the azimuthal angle $\phi$, connected to the structure of the input laser; this dependence will be discussed further in Sec. IV.

The angle dependence of the scattered intensity difference for CDS, CVDS, and their sum is illustrated in Fig. 3 for the indicative case of $\ell / k r=1$. Evidently, for the low value of $\ell / k r=1$, the CVDS contribution is seen to perturb the well-known CDS effect only slightly; note a shift of about 10 degrees in the angular minimum.

To investigate the effect of increasing the OAM of the incident laser beam, we may fix $k r=1$ and study the scatteringangle dependence of the scattered intensity difference for values of $\ell=1,5,10$, and 20, as exhibited in Fig. 4. Clearly evident is the substantial increase in magnitude of the scattered intensity difference for high values of $\ell$, and also

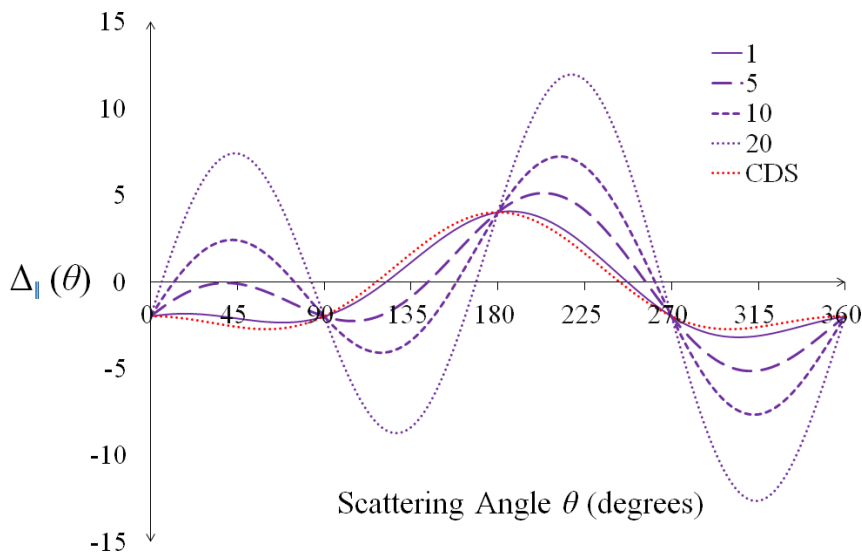

FIG. 4. Plot of differential scattered intensity of light with $\hat{\boldsymbol{k}} \hat{\boldsymbol{k}}^{\prime}$ plane polarization components against scattering angle $(k r=1)$. Varying the topological charge values highlights the scattered intensity difference enhancement for high values of $\ell$, and also shows how this changes for any given scattering angle. The shift in maximum scattering differences stems from a simple increase in the weighted contribution of the CVDS effect as $\ell$ increases. 




FIG. 5. Plot of differential scattered intensity of light with $\hat{\boldsymbol{k}} \hat{\boldsymbol{k}}^{\prime}$ plane polarization components against scattering angle. Varying the sign of topological charge values highlights the chiroptical effect due to the sign of $\ell$.

the corresponding shift in maximum scattering angle as the CVDS contribution becomes more heavily weighted at high $\ell$ compared to the standard CDS. As such, for high values of $\ell$ and a judiciously placed polarization analyzer, the optical activity signal can be significantly enhanced compared to that of CDS experiments where the laser beam possesses no optical OAM (i.e., the currently used form of light used in experiments). This is an important consequence, and a beneficial aspect of using structured light to enhance signals in chiral spectroscopy applications, as conventional optical activity effects are inherently weak due to their dependence on M1 and E2 interactions with the field.

Another important result from using twisted light beams is that they add a further chiroptical effect (or influence) to Rayleigh and Raman optical activity. The CVDS effect is dependent not only on the magnitude of $\ell$, but also its sign. The plot in Fig. 5 exhibits this chiroptical effect by highlighting how for the given positive values of $\ell=1,5,10$, and 20, the corresponding negative values give a different (or discriminatory) differential scattering intensity (the negative to be more specific). This is therefore a vortex analog to how standard CDS works with circular polarizations: in standard CDS, the differential scattering rate arises due to a difference in the scattering of right- and left-handed circularly polarized photons; in CVDS this is also the case, alongside the fact that the signal also differs for a right-handed vortex versus a left-handed vortex. If we denote the helicity of light as $\sigma= \pm 1$ for circular polarizations, we can see that this effect follows the simple relationships that it is invariant under the transformation $(\sigma, \ell) \rightarrow(-\sigma,-\ell)$, but not $(\sigma, \ell) \rightarrow(-\sigma, \ell)$ or $(\sigma, \ell) \rightarrow$ $(\sigma,-\ell)$, or alternatively denoting right-handedness as " $\mathrm{R}$ " and left-handedness as "L," then $\mathrm{LL}=\mathrm{RR} \neq \mathrm{LR}=\mathrm{RL}$. This mirrors a symmetry principle first established for circular vortex dichroism [26,27].

Experimentally, CDS is often observed by using circular polarization modulation, switching rapidly between $\sigma$ and $-\sigma$ (i.e., the handedness of the polarization) [67]. Very recently a device that can modulate the OAM of a laser continuously from $\ell$ to $-\ell$ at high speed has been produced [68]. By fixing the incident circular polarization of light, using such a device would therefore allow for the topological charge modulation analog of circular polarization used in standard CDS experiments for the CVDS effect.

\section{OBSERVING CVDS}

In Sec. III we looked at how the scattering angle and the OAM of the input beam influence the CVDS effect. We now develop a more detailed appraisal of point (e) from the list in Sec. III, specifically focusing on the fact that the CVDS effect has a $\cos \phi$ dependence, which the standard CDS does not. It is well known that in both Cartesian and cylindrical coordinate systems, the coordinate $z$ has an equivalent role in both; as such, any property that depends on $\hat{z}$ through an axial gradient (via an E2 interaction with the field) takes on a form for vortex light similar to the form it would take for a standard laser beam without OAM, both in the paraxial and nonparaxial regime (though in the latter there are subtle differences). This is why, alongside the novel CVDS effect due to the helical-phase structure, our methodology always permits recovery of the corresponding results for standard, well-known CDS. However, as we have seen, quadrupole transitions can also be driven by gradients in the transverse plane (specifically, helical-phase gradients that do not exist in light without OAM); for cylindrical coordinates these gradients depend on $\hat{\boldsymbol{r}}$ and $\hat{\boldsymbol{\phi}}$. Significantly, these do not follow such a simple coordinate transformation into Cartesian coordinates: $\hat{\boldsymbol{r}}=\hat{\boldsymbol{x}} \cos \phi+\hat{\boldsymbol{y}} \sin \phi ; \hat{\boldsymbol{\phi}}=-\hat{\boldsymbol{x}} \sin \phi+\hat{\boldsymbol{y}} \cos \phi$. The cylindrical symmetry of OAM modes therefore dictates that any optical interaction dependent linearly on either $\hat{\boldsymbol{r}}$ or $\hat{\boldsymbol{\phi}}$ should vanish upon a total integration over the transverse beam profile. Indeed, this is what is observed with the CVDS effect, simply stemming from $\langle\hat{\boldsymbol{\phi}}\rangle=\int_{0}^{2 \pi} \cos \phi d \phi=[\sin \phi]_{0}^{2 \pi}=0$. Trivially, this tells us then that when Rayleigh or Raman scattering is detected from the whole volume of the sample irradiated by the input beam, then the CVDS result vanishes, leaving only the standard CDS effect. This therefore means that in order to observe CVDS we require a simple off-axis beam alignment, where the maximum effect would occur for half of the beam being incident on the sample, or alternatively focusing elements in the detection arm are aligned to intercept local sections of the laser beam [69].

The $\hat{\boldsymbol{\phi}}$ terms are only present in beams with a helical-phase structure, and this phase structure rotates uniformly about the beam axis (rather than at individual points in space; see Fig. 6). Thus it is to always be anticipated that any chiroptical effect stemming from the gradients of transverse phase structure that depend linearly on $\ell$ vanish upon integration over the total beam profile - though axial phase gradient effects will still persist and relate to well-known optical activity phenomena, in this particular case CDS. Physically this can be seen to be a consequence of the fact that the OAM is a global (or spatial) property of the beam, whereas SAM (and circular polarization) is a local property. Interestingly, it is well known that the total angular momentum of light can manifest disparate characteristics stemming from its constituent SAM and OAM contributions. For example, the difference of rotation characteristics of light's SAM and OAM are most beautifully revealed in their mechanical effects on a particle: SAM causes the particle to spin around its own center of mass, while OAM causes the particle to orbit around the 

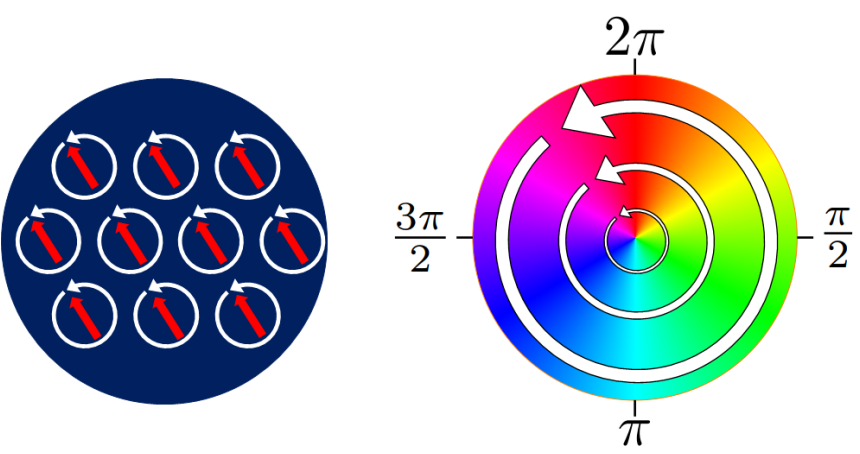

FIG. 6. Rotational transformations of the electromagnetic fields: left is a local rotation where the electric field vector (red arrow) at every position is rotated (SAM); right is a global rotation of the whole complex-amplitude structure of a twisted beam (OAM) around a given axis ( $z$ in our case). The SAM rotates around every point in the beam; the OAM rotates around the beam axis. Both correspond to left-handed forms of circularly polarized light and twisted light, respectively (figure adapted from $[74,75]$ ).

beam axis [70-72] (though a circularly polarized beam with an inhomogeneous intensity can itself cause an orbital motion of particles [73]). Here we have described disparate characteristics of light's total angular momentum in a spectroscopic application.

We now finally comment on a remaining issue with regard to practical strategies for observing CVDS. There is no doubt that the intricate dependence of the intensity profile [through the radial distribution function $f_{|\ell|, p}(r)$ ] and radius $R_{\ell}=w_{0} \sqrt{|\ell| / 2}$ of a Laguerre-Gaussian beam on $\ell$ suggests that quantitative analysis in spectroscopic applications may be complicated. First, for any given value of $\ell$, the $(k r)^{-1}$ dependence requires further thought in the pursuit of such quantitative predictions. Evidently, chiral particles positioned closer to the beam axis will contribute more significantly to the CVDS effect, but will equally experience a smaller intensity of light. However, as already discussed, this is to be expected as quadrupole transitions are driven by gradients, both axial and transverse. Experimentally, to offset the effects of a weaker intensity, we may increase the input beam intensity, use shorter-wavelength light, or utilize a more focused beam with a smaller beam waist. Of course, in order to observe the pure OAM effects described in this work, the beam should not be so focused as to cause appreciable spin-orbit interactions of light [29]. At high values of $\ell$ individual molecules close to the beam center (and therefore close to the axial phase singularity) might receive a negligible input intensity. This is a factor that might impinge negatively on securing CVDS signals from isolated regions of a heterogeneous sample, for example. To this end, in order to compensate for the broader beam spread, and corresponding reduction in power per unit area, it would be possible to apply appropriately higher levels of overall input intensity without the risk of competition from optically nonlinear effects that might normally intervene with a focused beam.

The $\ell$-dependent intensity distributions and beam profile width therefore make the CVDS effect and CID difficult to compare between any two different values of OAM of the incident beam. However, the recent development of so-called "perfect" optical vortices [76-80] could play an extremely important role in experimentally quantifying CVDS, and potentially any twisted light-matter spectroscopic technique. These perfect vortices possess an intensity profile and radius that are independent of $\ell$, but importantly their helical-phase structure still depends on $\ell$. This therefore enables the study of the twisted beam coupling to matter for varying values of $\ell$ without having to account for a varying intensity structure. Such an input should most readily allow for the utilization and exploitation of the linear scaling of CVDS with $\ell$, yielding significantly enhanced chiroptical spectroscopic signals as discussed in Sec. III. Indeed, perfect optical vortices have been experimentally produced that exhibit values of $\ell=90$ [81]. Evidently, the number and variety of experimentally controllable parameters that influence the observable CVDS and scattered intensities offer a rich scope of interplay in potential experiments pursuing the enhancement of optical activity signals with structured light.

\section{DISCUSSION AND SUMMARY}

In this paper we have derived and secured tractable results for the newly discovered circular-vortex differential scattering effect. Our formalism has allowed us to elicit many novel aspects of the effect (a list can be found in Sec. III). In particular, we have seen its unique scattering-angle dependence in comparison to the well-known circular differential scattering Rayleigh and Raman optical activity. Also, the analysis has shown how a dependence on the sign and magnitude of the OAM conveyed by the incident structured light beam offers the ability to significantly enhance the optical activity signals, appreciably beyond those possible with the current techniques based on laser beams with zero OAM. We would also like to draw attention to the fact the recently discovered CVD effect $[26,27]$ will also exhibit some of the important properties of the CVDS discussed in this paper, such as producing enhanced optical activity signals with an increase in input laser OAM. Furthermore, we have highlighted the distinct role that perfect optical vortices will have in the pursuit of observing and quantifying these enhanced optical activity signals.

To date, there have been few studies of the spectroscopic application of twisted light, with work in the nascent area generally concerned with answering fundamental questions such as whether optical OAM could be involved in atomic electronic transitions [82,83]. Interestingly, a few exploratory studies on spectroscopic applications of structured light possessing OAM and optical activity have indicated that such a chiroptical effect is possible [84,85], and the same group has recently published work where recognizable chiroptical effects occur in the transmission of vortex beams though mouse brain tissue [86]. From the analysis undertaken in this paper, it is clear that the application of twisted light in spectroscopy, particularly chiral spectroscopy, offers much additional scope for potentially unique applications in molecular systems, exploiting the more complicated and intricate structures of the OAM-bearing laser beam. Chiroptical spectroscopic techniques are becoming increasingly more important as the level of analysis is improving due to rapid advances 
in computational chemistry $[49,87,88]$, and the CVDS effect clearly has an important role to play in these spectroscopies, and especially vibrational optical activity. Future work will aim to develop the theory for ROA with structured light, beyond incident circular polarizations as described here, to address the potential novelties of resolving circular components of the Raman signal, and also dual circular polarization experiments [89].

\section{ACKNOWLEDGMENTS}

K.A.F. would like to thank the Leverhulme Trust for funding him through a Leverhulme Early Career Fellowship.

\section{APPENDIX}

First we highlight the derivation of Eq. (2.4) from the main article, taking the matrix element for the process derived in Ref. [28] (the factor of $1 / 2$ difference stems from a different definition of the normalization constant):

$$
M_{f i}=-\left(\frac{\hbar c}{2 \varepsilon_{0} V}\right) \sqrt{k k^{\prime} n} f_{|\ell|, p}(r) \bar{e}_{i}^{\prime} e_{k}\left\{\alpha_{i k}^{m 0}+\left(\frac{i \ell \hat{\phi}_{l}}{r}+i k \hat{z}_{l}+\hat{r}_{l}\left[f_{|\ell|, p}^{-1}(r) \partial_{r} f_{|\ell|, p}(r)-r^{-1}\right]\right) A_{i k l}^{m 0}-i k^{\prime}{ }_{l} A_{k i l}^{m 0}\right\} e^{-i k^{\prime} \cdot r} e^{i k z} e^{i \ell \phi} .
$$

The scattered radiant intensity of light per molecule is given by [2]

Inserting (A1) into (A2) produces

$$
I\left(\boldsymbol{k}^{\prime}\right)=\frac{k^{\prime 3} V}{4 \pi^{2} \hbar}\left|M_{f i}\right|^{2}
$$

$$
I\left(\boldsymbol{k}^{\prime}\right)=\left[\frac{n \hbar c^{2} k}{V} f_{|\ell|, p}^{2}(r)\right] \frac{k^{\prime 4}}{8 \pi^{2} \varepsilon_{0}^{2}}\left|\bar{e}_{i}^{\prime} e_{k}\left\{\alpha_{i k}^{m 0}+\left(\frac{i \ell \hat{\phi}_{l}}{r}+i k \hat{z}_{l}+\hat{r}_{l}\left[f_{|\ell|, p}^{-1}(r) \partial_{r} f_{|\ell|, p}(r)-r^{-1}\right]\right) A_{i k l}^{m 0}-i k^{\prime} A_{k i l}^{m 0}\right\}\right|^{2},
$$

where the first term in square brackets on the right-hand side is the irradiance of the input light, i.e., the incident power per unit area $I_{0}(r)=n \hbar c^{2} k f_{|\ell|, p}^{2}(r) / V$. We now look to carry out the modulus square term in (A3) and retaining only the $\alpha A$ terms, which yields

$$
I^{\alpha A}\left(\boldsymbol{k}^{\prime}\right)=-\frac{I_{0}(r) k^{\prime 4}}{8 \pi^{2} \varepsilon_{0}^{2}} \bar{e}_{i}^{\prime} e_{j}^{\prime} e_{k} \bar{e}_{m}\left[\left(\frac{i \ell \hat{\phi}_{l}}{r}+i k \hat{z}_{l}+\hat{r}_{l}\left[f_{|\ell|, p}^{-1}(r) \partial_{r} f_{|\ell|, p}(r)-r^{-1}\right]\right) \alpha_{i k}^{m 0} A_{j m l}^{m 0}-i k_{l}^{\prime} \alpha_{i k}^{m 0} A_{m j l}^{m 0}\right] .
$$

As it stands (A4) is applicable to systems with oriented molecules - a solid, for example. By accounting for molecular tumbling through a fully three-dimensional rotational average of the molecular factors in (A4), we may derive a formula applicable to the more pertinent case in optical activity studies of an isotropic sample, such as a liquid. Using standard techniques [63], the fifth-rank full rotational tensor average required for (A4) is calculated via

$$
\begin{aligned}
I\left(\boldsymbol{k}^{\prime}\right)= & -\frac{I_{0}(r) k^{\prime 4}}{8 \pi^{2} \varepsilon_{0}^{2}}\left\langle\overline { e } _ { i } ^ { \prime } e ^ { \prime } { } _ { j } e _ { k } \overline { e } _ { m } \left[\left(\frac{i \ell \hat{\phi}_{l}}{r}+i k \hat{z}_{l}+\hat{r}_{l}\left[f_{|\ell|, p}^{-1}(r) \partial_{r} f_{|\ell|, p}(r)-r^{-1}\right]\right)\left\langle R_{i \lambda} R_{k \mu} R_{j v} R_{m o} R_{l \pi}\right\rangle\right.\right. \\
& \left.\left.-i k^{\prime}{ }_{l}\left\langle R_{i \lambda} R_{k \mu} R_{m \nu} R_{j o} R_{l \pi}\right\rangle\right] \alpha_{\lambda \mu}^{m 0} A_{v o \pi}^{m 0}\right\rangle,
\end{aligned}
$$

where angular brackets denote the average, Latin indices are related to space-fixed frames, and Greek to molecule-fixes frames, and

$$
\left\langle R_{i \lambda} R_{k \mu} R_{j v} R_{m o} R_{l \pi}\right\rangle=\frac{1}{30}\left(\varepsilon_{i j m} \delta_{k l} \varepsilon_{\lambda \nu o} \delta_{\mu \pi}+\varepsilon_{i j l} \delta_{k m} \varepsilon_{\lambda \nu \pi} \delta_{\mu o}+\varepsilon_{k j m} \delta_{i l} \varepsilon_{\mu \nu o} \delta_{\lambda \pi}+\varepsilon_{k j l} \delta_{i m} \varepsilon_{\mu \nu \pi} \delta_{\lambda o}\right) .
$$

The average (A6) is a shorter version than usually quoted for fifth-rank tensor averaging because in this case we may utilize the fact that the pairs of indices $l, k$ and $m, l$ are index-symmetric along with the fact that the quadrupole moment is traceless, i.e., $A_{i j j}=0$. Furthermore, Eq. (A6) specifically relates to the first average in angular brackets on the right-hand side of (A5), but the simple transformation of $m \leftrightarrow j$ gives the correct form necessary to carry out the other average in (A5). There are twelve individual tensor contractions and contributions to the total scattered intensity (A5) to undertake in the total average; an indicative one $I^{\prime}\left(\boldsymbol{k}^{\prime}\right)$ is

$$
\begin{aligned}
I^{\prime}\left(\boldsymbol{k}^{\prime}\right) & =-\frac{I_{0}(r) k^{\prime 4}}{8 \pi^{2} \varepsilon_{0}^{2}} i k \frac{1}{30} \bar{e}_{i}^{\prime} e_{j}^{\prime} e_{k} \bar{e}_{m} \hat{z}_{l} \varepsilon_{k j m} \delta_{i l} \varepsilon_{\mu \nu o} \delta_{\lambda \pi} \alpha_{\lambda \mu}^{m 0} A_{\nu o \pi}^{m 0} \\
& =-\frac{I_{0}(r) k^{\prime 4}}{8 \pi^{2} \varepsilon_{0}^{2}} i k \frac{1}{30}\left(\hat{z} \cdot \overline{\boldsymbol{e}}^{\prime}\right)(\overline{\boldsymbol{e}} \times \boldsymbol{e}) \cdot \boldsymbol{e}^{\prime} \alpha_{\lambda \pi}^{m 0} A_{\mu \pi}^{\prime m 0} \\
& =\frac{I_{0}(r) k^{\prime 4}}{240 \pi^{2} \varepsilon_{0}^{2}} i k\left(\hat{z} \cdot \overline{\boldsymbol{e}}^{\prime}\right)\left(\boldsymbol{c} \cdot \boldsymbol{e}^{\prime}\right) \alpha_{\lambda \pi}^{m 0} A_{\mu \pi}^{\prime m 0},
\end{aligned}
$$


where $\boldsymbol{c}=\boldsymbol{e} \times \overline{\boldsymbol{e}}$. Carrying out all the tensor contractions, noting the orthogonal nature of $(\hat{\boldsymbol{k}} \cdot \boldsymbol{e})=0,\left(\hat{\boldsymbol{k}}^{\prime} \cdot \boldsymbol{e}^{\prime}\right)=0$, and that for scattered in-plane $c^{\prime}(\|)=0$ and transverse polarizations $c^{\prime}(\perp)=0$, gives the result (2.4) from the main article:

$$
\begin{aligned}
I^{\alpha A}\left(\boldsymbol{k}^{\prime}\right)= & \beta i k\left\{\left(\hat{\boldsymbol{k}}^{\prime} \cdot \boldsymbol{c}\right)+\left(\hat{z} \cdot \overline{\boldsymbol{e}}^{\prime}\right)\left(\boldsymbol{c} \cdot \boldsymbol{e}^{\prime}\right)+\left(\overline{\boldsymbol{b}^{\prime}} \cdot \overline{\boldsymbol{e}}\right)\left(\boldsymbol{e} \cdot \boldsymbol{e}^{\prime}\right)-\left(\boldsymbol{b} \cdot \boldsymbol{e}^{\prime}\right)\left(\overline{\boldsymbol{e}} \cdot \overline{\boldsymbol{e}^{\prime}}\right)-i k^{-1}\left[f_{|\ell|, p}^{-1}(r) \partial_{r} f_{|\ell|, p}(r)-r^{-1}\right]\right. \\
& \left.\times\left[\left(\hat{\boldsymbol{r}} \cdot \overline{\boldsymbol{e}}^{\prime}\right)\left(\boldsymbol{c} \cdot \boldsymbol{e}^{\prime}\right)-\left(\hat{\boldsymbol{r}} \times \boldsymbol{e} \cdot \boldsymbol{e}^{\prime}\right)\left(\overline{\boldsymbol{e}} \cdot \overline{\boldsymbol{e}^{\prime}}\right)\right]+\ell k^{-1} r^{-1}\left(\hat{\boldsymbol{\phi}} \cdot \overline{\boldsymbol{e}}^{\prime}\right)\left(\boldsymbol{c} \cdot \boldsymbol{e}^{\prime}\right)-\ell k^{-1} r^{-1}\left(\hat{\boldsymbol{\phi}} \times \boldsymbol{e} \cdot \boldsymbol{e}^{\prime}\right)\left(\overline{\boldsymbol{e}} \cdot \overline{\boldsymbol{e}^{\prime}}\right)\right\} \alpha_{\lambda \mu}^{m 0} A_{\lambda \mu}^{\prime m 0},
\end{aligned}
$$

where we have used $A_{\lambda \mu}^{\prime m 0}=\varepsilon_{\lambda \alpha \beta} A_{\alpha \beta \mu}^{m 0}$ and $\beta=I_{0}(r) k^{4} / 240 \varepsilon_{0}^{2} \pi^{2}$. We have also used $k=k^{\prime}$ which is correct for Rayleigh scattering, and an approximation for Raman scattering, justified because vibrational energy differences are usually very small compared to the electronic energies featured in the molecular response tensors.

Analogous results follow similar lines of derivation for $\alpha^{2}$ scattering if only the first term dependent on $\alpha_{i k}^{m 0}$ is retained in (A1), while the necessary matrix element to derive $\alpha G$ contributions to scattering is

$$
M_{f i}=-\left(\frac{\hbar}{4 \varepsilon_{0} V}\right) \sqrt{k k^{\prime} n} f_{|\ell|, p}(r)\left(c \bar{e}_{i}^{\prime} e_{k} \alpha_{i k}^{m 0}+\bar{e}_{i}^{\prime} b_{k} G_{i k}^{m 0}+\bar{b}_{i}^{\prime} e_{k} \bar{G}_{k i}^{m 0}\right) e^{-i \boldsymbol{k}^{\prime} \cdot \boldsymbol{r}} e^{i k z} e^{i \ell \phi} .
$$

The ensuing orientational averages are of fourth rank, in comparison to the fifth-rank average (A6).

In order to develop our scattered intensities into a circular intensity differential ratio, where the scattered radiation has either in-plane or transverse polarization, for an arbitrary scattering angle involves numerous calculations. To exhibit the methods we will explicitly highlight the calculations for the $\alpha A$ case of key interest, and simply report the other results.

We first calculate the scattered intensity of input right-handed circularly polarized $(\mathrm{R})$ into in-plane polarized scattered light $I^{(\alpha A)}(\mathrm{R} \rightarrow \|)$. Carrying out the vector products for each term in (A8) and using the scattering geometry exhibited in Fig. 2 of the main article,

$$
\begin{gathered}
i\left(\hat{\boldsymbol{k}}^{\prime} \cdot \boldsymbol{c}^{\mathrm{R}}\right)=i\left(\hat{\boldsymbol{k}}^{\prime} \cdot i \hat{z}\right)=-\cos \theta, \\
i\left(\hat{\boldsymbol{z}} \cdot \overline{\boldsymbol{e}}_{(\|)}^{\prime}\right)\left(\boldsymbol{c}^{\mathrm{R}} \cdot \boldsymbol{e}_{(\|)}^{\prime}\right)=i^{2}\left(\hat{z} \cdot \overline{\boldsymbol{e}}_{(\|)}^{\prime}\right)\left(\hat{z} \cdot \boldsymbol{e}_{(\|)}^{\prime}\right)=-\sin ^{2} \theta=\cos ^{2} \theta-1,
\end{gathered}
$$

where we have used $\boldsymbol{e}^{\mathrm{L} / \mathrm{R}}=\frac{1}{\sqrt{2}}\left(\boldsymbol{e}_{(\|)} \pm i \boldsymbol{e}_{(\perp)}\right)$ and thus $\boldsymbol{c}^{\mathrm{L} / \mathrm{R}}=\boldsymbol{e}^{\mathrm{L} / \mathrm{R}} \times \overline{\boldsymbol{e}}^{\mathrm{L} / \mathrm{R}}=\mp i \hat{\boldsymbol{k}}=\mp i \hat{z}$.

$$
\begin{aligned}
i\left(\overline{\boldsymbol{b}}_{(\|)}^{\prime} \cdot \overline{\boldsymbol{e}}^{\mathrm{R}}\right)\left(\boldsymbol{e}^{\mathrm{R}} \cdot \boldsymbol{e}_{(\|)}^{\prime}\right) & =i\left\{\overline{\boldsymbol{e}}_{(\perp)} \cdot\left[\frac{1}{\sqrt{2}}\left(\boldsymbol{e}_{(\|)}+i \boldsymbol{e}_{(\perp)}\right)\right]\right\}\left\{\left[\frac{1}{\sqrt{2}}\left(\boldsymbol{e}_{(\|)}-i \boldsymbol{e}_{(\perp)}\right)\right] \cdot \boldsymbol{e}_{(\|)}^{\prime}\right\} \\
& =\frac{i}{2}\left[\left(\overline{\boldsymbol{e}}_{(\perp)}^{\prime} \cdot \boldsymbol{e}_{(\|)}+i \boldsymbol{e}_{(\perp)} \cdot \overline{\boldsymbol{e}}_{(\perp)}\right)\left(\boldsymbol{e}_{(\|)}^{\prime} \cdot \boldsymbol{e}_{(\|)}-i \boldsymbol{e}_{(\perp)} \cdot \boldsymbol{e}_{(\|)}^{\prime}\right)\right] \\
& =-\frac{1}{2} \boldsymbol{e}_{(\|)}^{\prime} \cdot \boldsymbol{e}_{(\|)}=-\frac{1}{2} \cos \theta,
\end{aligned}
$$

exploiting the fact that $\hat{\boldsymbol{k}} \times \hat{\boldsymbol{e}}=\hat{\boldsymbol{b}}$.

$$
\begin{aligned}
-i\left(\boldsymbol{b}^{\mathrm{R}} \cdot \boldsymbol{e}^{\prime}(\|)\right)\left(\overline{\boldsymbol{e}}^{\mathrm{R}} \cdot \overline{\boldsymbol{e}}_{(\|)}^{\prime}\right) & =-i\left(i \boldsymbol{e}^{\mathrm{R}} \cdot \boldsymbol{e}^{\prime}{ }_{(\|)}\right)\left(\overline{\boldsymbol{e}}^{\mathrm{R}} \cdot \overline{\boldsymbol{e}}_{(\|)}\right) \\
& =-i^{2}\left(\left\{\left[\frac{1}{\sqrt{2}}\left(\boldsymbol{e}_{(\|)}-i_{(\perp)}\right)\right] \cdot \boldsymbol{e}_{(\|)}^{\prime}\right\}\left\{\left[\frac{1}{\sqrt{2}}\left(\boldsymbol{e}_{(\|)}+i \boldsymbol{e}_{(\perp)}\right)\right] \cdot \overline{\boldsymbol{e}}_{(\|)}\right\}\right) \\
& =\frac{1}{2}\left(\boldsymbol{e}_{(\|)}^{\prime} \cdot \boldsymbol{e}_{(\|)}\right)\left(\overline{\boldsymbol{e}}_{(\|)} \cdot \boldsymbol{e}_{(\|)}\right)=\frac{1}{2} \cos ^{2} \theta
\end{aligned}
$$

given the identity $\hat{\boldsymbol{b}}^{(\mathrm{L} / \mathrm{R})}=\mp i \hat{\boldsymbol{e}}^{(\mathrm{L} / \mathrm{R})}$.

$$
\begin{aligned}
\frac{i \ell}{k r}\left(\hat{\boldsymbol{\phi}} \cdot \overline{\boldsymbol{e}}_{(\|)}^{\prime}\right)\left(\boldsymbol{c}^{\mathrm{R}} \cdot \boldsymbol{e}_{(\|)}^{\prime}\right) & =\frac{i \ell}{k r}\left[(-\hat{\boldsymbol{x}} \sin \phi+\hat{\boldsymbol{y}} \cos \phi) \cdot \overline{\boldsymbol{e}}_{\left(\| / \hat{\boldsymbol{y}}^{\prime}\right)}^{\prime}\right]\left(i \hat{\boldsymbol{z}} \cdot \boldsymbol{e}_{\left(\| / \hat{\boldsymbol{y}}^{\prime}\right)}^{\prime}\right) \\
& =-\frac{\ell}{k r}\left(\overline{\boldsymbol{e}}_{\left(\| / \hat{\boldsymbol{y}}^{\prime}\right)}^{\prime} \cdot \hat{\boldsymbol{y}} \cos \phi\right)\left(\hat{\boldsymbol{z}} \cdot \boldsymbol{e}_{\left(\| / \hat{\boldsymbol{y}}^{\prime}\right)}^{\prime}\right) \\
& =\frac{\ell}{k r} \sin \theta \cos \theta \cos \phi=\frac{\ell}{2 k r} \sin 2 \theta \cos \phi,
\end{aligned}
$$

utilizing the well-known coordinate transformation $\hat{\boldsymbol{\phi}}=-\hat{\boldsymbol{x}} \sin \phi+\hat{\boldsymbol{y}} \cos \phi$, and well-known trigonometric identities.

$$
\begin{aligned}
& -\frac{i \ell}{k r}\left[\left(\hat{\boldsymbol{\phi}} \times \boldsymbol{e}^{\mathrm{R}}\right) \cdot \boldsymbol{e}_{(\|)}^{\prime}\right]\left(\overline{\boldsymbol{e}}^{\mathrm{R}} \cdot \overline{\boldsymbol{e}}_{(\|)}^{\prime}\right)=\frac{i \ell}{k r}\left\{(\hat{\boldsymbol{x}} \sin \phi+\hat{\boldsymbol{y}} \cos \phi) \times\left[\frac{1}{\sqrt{2}}\left(\boldsymbol{e}_{(\|)}-i \boldsymbol{e}_{(\perp)}\right)\right] \cdot \boldsymbol{e}^{\prime}{ }_{\left(\| / \hat{\boldsymbol{y}}^{\prime}\right)}\right\}\left(\overline{\boldsymbol{e}}^{\mathrm{R}} \cdot \overline{\boldsymbol{e}}_{\left(\| / \hat{\boldsymbol{y}}^{\prime}\right)}\right) \\
& =\frac{i \ell}{\sqrt{2} k r}\left[(-\hat{z} \sin \phi-i \hat{z} \cos \phi) \cdot \boldsymbol{e}_{\left(\| / \hat{y}^{\prime}\right)}^{\prime}\left[\frac{1}{\sqrt{2}}\left(\boldsymbol{e}_{(\|)}+i \boldsymbol{e}_{(\perp)}\right) \cdot \overline{\boldsymbol{e}}_{\left(\| / \hat{\boldsymbol{y}}^{\prime}\right)}\right]\right.
\end{aligned}
$$




$$
\begin{aligned}
& =\operatorname{Re} \frac{i \ell}{2 k r}(\sin \theta \sin \phi+i \sin \theta \cos \phi) \cos \theta \\
& =-\frac{\ell}{4 k r} \sin 2 \theta \cos \phi .
\end{aligned}
$$

Using the fact that $I^{(\alpha A)}(\mathrm{R} \rightarrow \|)=-I^{(\alpha A)}(\mathrm{L} \rightarrow \|)$ now allows us to write

$$
\Delta I^{\alpha A}\left(\boldsymbol{k}^{\prime}, \|\right)=\beta k\left[3 \cos ^{2} \theta-3 \cos \theta-2+\frac{\ell}{2 k r} \sin 2 \theta \cos \phi\right] \alpha_{\lambda \mu}^{m 0} A_{\lambda \mu}^{m 0} .
$$

We now calculate the scattered intensity of light with transverse polarization $I^{(\alpha A)}(\mathrm{R} \rightarrow \perp)$ :

$$
\begin{aligned}
& i\left(\hat{\boldsymbol{k}}^{\prime} \cdot \boldsymbol{c}^{\mathrm{R}}\right)=i\left(\hat{\boldsymbol{k}}^{\prime} \cdot i \hat{\boldsymbol{z}}\right)=-\cos \theta, \\
& i\left(\hat{z} \cdot \overline{\boldsymbol{e}}_{(\perp)}^{\prime}\right)\left(\boldsymbol{c}^{\mathrm{R}} \cdot \boldsymbol{e}^{\prime}(\perp)=0,\right. \\
& i\left(\overline{\boldsymbol{b}}_{(\perp)}^{\prime} \cdot \overline{\boldsymbol{e}}^{\mathrm{R}}\right)\left(\boldsymbol{e}^{\mathrm{R}} \cdot \boldsymbol{e}^{\prime}{ }_{(\perp)}\right)=i\left\{-\overline{\boldsymbol{e}}_{(\|)} \cdot\left[\frac{1}{\sqrt{2}}\left(\boldsymbol{e}_{(\|)}+i \boldsymbol{e}_{(\perp)}\right)\right]\right\}\left\{\left[\frac{1}{\sqrt{2}}\left(\boldsymbol{e}_{(\|)}-i \boldsymbol{e}_{(\perp)}\right)\right] \cdot \boldsymbol{e}^{\prime}{ }_{(\perp)}\right\} \\
& =-\frac{i}{2}\left[\left(\overline{\boldsymbol{e}}_{(\|)}^{\prime} \cdot \boldsymbol{e}_{(\|)}+i \boldsymbol{e}_{(\perp)} \cdot \overline{\boldsymbol{e}}_{(\|)}\right)\left(\boldsymbol{e}_{(\|)} \cdot \boldsymbol{e}_{(\perp)}^{\prime}-i \boldsymbol{e}_{(\perp)} \cdot \boldsymbol{e}_{(\perp)}^{\prime}\right)\right] \\
& =-\frac{1}{2} \overline{\boldsymbol{e}}_{(\|)} \cdot \boldsymbol{e}_{(\|)}=-\frac{1}{2} \cos \theta, \\
& -i\left(\boldsymbol{b}^{\mathrm{R}} \cdot \boldsymbol{e}_{(\perp)}^{\prime}\right)\left(\overline{\boldsymbol{e}}^{\mathrm{R}} \cdot \overline{\boldsymbol{e}}_{(\perp)}^{\prime}\right)=-i\left(i \boldsymbol{e}^{\mathrm{R}} \cdot \boldsymbol{e}_{(\perp)}^{\prime}\right)\left(\overline{\boldsymbol{e}}^{\mathrm{R}} \cdot \overline{\boldsymbol{e}}_{(\perp)}^{\prime}\right) \\
& =-i^{2}\left(\left\{\left[\frac{1}{\sqrt{2}}\left(\boldsymbol{e}_{(\|)}-i \boldsymbol{e}_{(\perp)}\right)\right] \cdot \boldsymbol{e}_{(\perp)}^{\prime}\right\}\left\{\left[\frac{1}{\sqrt{2}}\left(\boldsymbol{e}_{(\|)}+i \boldsymbol{e}_{(\perp)}\right)\right] \cdot \overline{\boldsymbol{e}}_{(\perp)}\right\}\right) \\
& =\frac{1}{2}\left(\boldsymbol{e}_{(\perp)}^{\prime} \cdot \boldsymbol{e}_{(\perp)}\right)\left(\overline{\boldsymbol{e}}_{(\perp)}^{\prime} \cdot \boldsymbol{e}_{(\perp)}\right)=\frac{1}{2}, \\
& \frac{i \ell}{k r}\left(\hat{\boldsymbol{\phi}} \cdot \overline{\boldsymbol{e}}_{(\perp)}^{\prime}\right)\left(\boldsymbol{c}^{\mathrm{R}} \cdot \boldsymbol{e}^{\prime}{ }_{(\perp)}\right)=\frac{i \ell}{k r}\left[(-\hat{\boldsymbol{x}} \sin \phi+\hat{\boldsymbol{y}} \cos \phi) \cdot \overline{\boldsymbol{e}}_{\left(\perp / \hat{\boldsymbol{x}}^{\prime}\right)}^{\prime}\right]\left(i \hat{z} \cdot \boldsymbol{e}_{\left(\perp / \hat{\boldsymbol{x}}^{\prime}\right)}^{\prime}\right)=0,
\end{aligned}
$$

where this contribution vanishes because of the final dot product on the right-hand side.

$$
\begin{aligned}
-\frac{i \ell}{k r}\left[\left(\hat{\boldsymbol{\phi}} \times \boldsymbol{e}^{\mathrm{R}}\right) \cdot \boldsymbol{e}_{(\perp)}^{\prime}\right]\left(\overline{\boldsymbol{e}}^{\mathrm{R}} \cdot \overline{\boldsymbol{e}}_{(\perp)}\right) & =\frac{i \ell}{k r}\left\{(\hat{\boldsymbol{x}} \sin \phi+\hat{\boldsymbol{y}} \cos \phi) \times\left[\frac{1}{\sqrt{2}}\left(\boldsymbol{e}_{(\|)}-i \boldsymbol{e}_{(\perp)}\right)\right] \cdot \boldsymbol{e}_{\left(\perp / \hat{\boldsymbol{x}}^{\prime}\right)}^{\prime}\right\}\left(\overline{\boldsymbol{e}}^{\mathrm{R}} \cdot \overline{\boldsymbol{e}}_{\left(\perp / \hat{\boldsymbol{x}}^{\prime}\right)}\right) \\
& =\frac{i \ell}{\sqrt{2} k r}\left[( - \hat { z } \operatorname { s i n } \phi - i \hat { z } \operatorname { c o s } \phi ) \cdot \boldsymbol { e } _ { ( \perp / \hat { \boldsymbol { x } } ^ { \prime } ) } ^ { \prime } \left[\left[\frac{1}{\sqrt{2}}\left(\boldsymbol{e}_{(\|)}+i \boldsymbol{e}_{(\perp)}\right) \cdot \boldsymbol{e}_{\left(\perp / \hat{\boldsymbol{x}}^{\prime}\right)}^{\prime}\right]=0 .\right.\right.
\end{aligned}
$$

This contribution vanishes because of the terms in the first pair of square brackets on the right-hand side. Understanding that $I^{(\alpha A)}(\mathrm{R} \rightarrow \perp)=-I^{(\alpha A)}(\mathrm{L} \rightarrow \perp)$, we obtain

$$
\Delta I^{\alpha A}\left(\boldsymbol{k}^{\prime}, \perp\right)=\beta k[1-3 \cos \theta] \alpha_{\lambda \mu}^{m 0} A_{\lambda \mu}^{m 0}
$$

The calculations for $I^{\left(\alpha^{2}\right)}(\mathrm{R} / \mathrm{L} \rightarrow \|), I^{(\alpha G)}(\mathrm{R} \rightarrow \|)$, and $I^{(\alpha G)}(\mathrm{L} \rightarrow \|)$ follow along similar lines, and are given as

$$
\begin{gathered}
I^{\alpha^{2}}\left(\boldsymbol{k}^{\prime}\right)(\mathrm{R} / \mathrm{L} \rightarrow \|)=\frac{\beta}{2}\left[\left(3 \cos ^{2} \theta-2\right) \alpha_{\lambda \lambda}^{m 0} \alpha_{\mu \mu}^{m 0}+\left(\cos ^{2} \theta+6\right) \alpha_{\lambda \mu}^{m 0} \alpha_{\lambda \mu}^{m 0}\right], \\
I^{\alpha G}\left(\boldsymbol{k}^{\prime}\right)(\mathrm{R} \rightarrow \|)=-I^{\alpha G}\left(\boldsymbol{k}^{\prime}\right)(\mathrm{L} \rightarrow \|) \\
=\frac{\beta}{c}\left[\left(3 \cos ^{2} \theta-5 \cos \theta-2\right) \alpha_{\lambda \lambda}^{m 0} G_{\mu \mu}^{m 0}+\left(\cos ^{2} \theta+5 \cos \theta+6\right) \alpha_{\lambda \mu}^{m 0} G_{\lambda \mu}^{m 0}\right], \\
I^{\alpha^{2}}\left(\boldsymbol{k}^{\prime}\right)(\mathrm{R} / \mathrm{L} \rightarrow \perp)=\frac{\beta}{2}\left[\alpha_{\lambda \lambda}^{m 0} \alpha_{\mu \mu}^{m 0}+7 \alpha_{\lambda \mu}^{m 0} \alpha_{\lambda \mu}^{m 0}\right],
\end{gathered}
$$

and

$$
\begin{aligned}
I^{\alpha G}\left(\boldsymbol{k}^{\prime}\right)(\mathrm{R} \rightarrow \perp) & =-I^{\alpha G}\left(\boldsymbol{k}^{\prime}\right)(\mathrm{L} \rightarrow \perp) \\
& =\frac{\beta}{c}\left[(1-5 \cos \theta) \alpha_{\lambda \lambda}^{m 0} G_{\mu \mu}^{m 0}+(5 \cos \theta+7) \alpha_{\lambda \mu}^{m 0} G_{\lambda \mu}^{m 0}\right]
\end{aligned}
$$

From these scattered intensity contributions and using (2.3) from the main article, the CID ratios (2.11) and (2.12) are found. 
$\hat{\boldsymbol{r}}$-dependent terms. Although we neglected the $\hat{\boldsymbol{r}}$ in (A8) in the article for reasons detailed there, we now finally give their contributions to the CID ratios as per above for completeness.

$$
\begin{aligned}
-C\left(\hat{\boldsymbol{r}} \times \boldsymbol{e}^{R} \cdot \boldsymbol{e}^{\prime}{ }_{(\|)}\right)\left(\overline{\boldsymbol{e}}^{R} \cdot \overline{\boldsymbol{e}}_{(\|)}^{\prime}\right) & =-C\left\{(\hat{\boldsymbol{x}} \cos \phi+\hat{\boldsymbol{y}} \sin \phi) \times\left[\frac{1}{\sqrt{2}}\left(\boldsymbol{e}_{(\|)}-i \boldsymbol{e}_{(\perp)}\right)\right] \cdot \boldsymbol{e}_{\left(\| / \hat{\boldsymbol{y}}^{\prime}\right)}^{\prime}\right\}\left(\overline{\boldsymbol{e}}^{\mathrm{R}} \cdot \overline{\boldsymbol{e}}_{\left(\| / \hat{\boldsymbol{y}}^{\prime}\right)}\right) \\
& =-C\left[( - \hat { z } \operatorname { c o s } \phi - i \hat { z } \operatorname { s i n } \phi ) \cdot \boldsymbol { e } _ { ( \| / \hat { \boldsymbol { y } } ^ { \prime } ) } ^ { \prime } \left[\left[\frac{1}{\sqrt{2}}\left(\boldsymbol{e}_{(\|)}+i \boldsymbol{e}_{(\perp)}\right) \cdot \overline{\boldsymbol{e}}_{\left(\| / \hat{\boldsymbol{y}}^{\prime}\right)}\right]\right.\right. \\
& =-C \operatorname{Re} \frac{1}{\sqrt{2}}(\sin \theta \cos \phi+i \sin \theta \sin \phi) \cos \theta \\
& =-C \frac{1}{2} \frac{1}{\sqrt{2}} \sin 2 \theta \cos \phi,
\end{aligned}
$$

where $C=\left[f_{|\ell|, p}^{-1}(r) \partial_{r} f_{|\ell|, p}(r)-r^{-1}\right]$. In this case $I^{(\alpha A)}(\mathrm{R} \rightarrow \|)=I^{(\alpha A)}(\mathrm{L} \rightarrow \|)$, and therefore the differential (but obviously not the sum) is zero (as expected because these terms are not discriminatory). For the case of $I^{(\alpha A)}(\mathrm{R} \rightarrow \perp)$,

$$
\begin{aligned}
-C\left(\hat{\boldsymbol{r}} \times \boldsymbol{e}^{\mathrm{R}} \cdot \boldsymbol{e}^{\prime}(\perp)\right)\left(\overline{\boldsymbol{e}}^{\mathrm{R}} \cdot \overline{\boldsymbol{e}}_{(\perp)}^{\prime}\right) & =-C\left\{(\hat{\boldsymbol{x}} \sin \phi+\hat{\boldsymbol{y}} \cos \phi) \times\left[\frac{1}{\sqrt{2}}\left(\boldsymbol{e}_{(\|)}-i \boldsymbol{e}_{(\perp)}\right)\right] \cdot \boldsymbol{e}_{\left(\perp / \hat{\boldsymbol{x}}^{\prime}\right)}^{\prime}\right\}\left(\overline{\boldsymbol{e}}^{\mathrm{R}} \cdot \overline{\boldsymbol{e}}_{\left(\perp / \hat{\boldsymbol{x}}^{\prime}\right)}\right) \\
& =-C \frac{1}{\sqrt{2}}\left[(-\hat{z} \cos \phi-i \hat{z} \sin \phi) \cdot \boldsymbol{e}_{\left(\perp / \hat{\boldsymbol{x}}^{\prime}\right)}^{\prime}\right]\left[\frac{1}{\sqrt{2}}\left(\boldsymbol{e}_{(\|)}+i \boldsymbol{e}_{(\perp)}\right) \cdot \boldsymbol{e}_{\left(\perp / \hat{x}^{\prime}\right)}^{\prime}\right]=0,
\end{aligned}
$$

where again $I^{(\alpha A)}(\mathrm{R} \rightarrow \perp)=I^{(\alpha A)}(\mathrm{L} \rightarrow \perp)$. Finally we have the terms

$$
C\left(\hat{\boldsymbol{r}} \cdot \overline{\boldsymbol{e}}^{\prime}\right)\left(\boldsymbol{c}^{\mathrm{R} / \mathrm{L}} \cdot \boldsymbol{e}^{\prime}\right)= \pm i C\left(\hat{\boldsymbol{r}} \cdot \overline{\boldsymbol{e}}^{\prime}\right)\left(\hat{\boldsymbol{k}} \cdot \boldsymbol{e}^{\prime}\right) .
$$

This contribution is imaginary and therefore taking the real part does not contribute to the observable rate of scattering.

[1] L. D. Barron, Molecular Light Scattering and Optical Activity (Cambridge University Press, Cambridge, 2009).

[2] D. P. Craig and T. Thirunamachandran, Molecular Quantum Electrodynamics: An Introduction to Radiation-Molecule Interactions (Courier Corporation, New York, 1998).

[3] D. L. Andrews, Quantum formulation for nanoscale optical and material chirality: Symmetry issues, space and time parity, and observables, J. Opt. 20, 033003 (2018).

[4] V. K. Valev, J. J. Baumberg, C. Sibilia, and T. Verbiest, Chirality and chiroptical effects in plasmonic nanostructures: Fundamentals, recent progress, and outlook, Adv. Mater. 25, 2517 (2013).

[5] P. Lodahl, S. Mahmoodian, S. Stobbe, A. Rauschenbeutel, P. Schneeweiss, J. Volz, H. Pichler, and P. Zoller, Chiral quantum optics, Nature (London) 541, 473 (2017).

[6] M. J. Urban, C. Shen, X.-T. Kong, C. Zhu, A. O. Govorov, Q. Wang, M. Hentschel, and N. Liu, Chiral plasmonic nanostructures enabled by bottom-up approaches, Annu. Rev. Phys. Chem. 70, 275 (2019).

[7] S. Nechayev and P. Banzer, Mimicking chiral light-matter interaction, Phys. Rev. B 99, 241101(R) (2019).

[8] K. Claborn, C. Isborn, W. Kaminsky, and B. Kahr, Optical rotation of achiral compounds, Angew. Chem. Int. Ed. 47, 5706 (2008).

[9] P. J. Stephens, Theory of magnetic circular dichroism, J. Chem. Phys. 52, 3489 (1970).

[10] W. P. Healy, The multipole Hamiltonian and magnetic circular dichroism, J. Chem. Phys. 64, 3111 (1976).

[11] T. Thirunamachandran, Theory of laser-induced optical activity, Proc. R. Soc. London, Ser. A 365, 327 (1979).

[12] L. Allen, M. W. Beijersbergen, R. J. C. Spreeuw, and J. P. Woerdman, Orbital angular momentum of light and the transformation of Laguerre-Gaussian laser modes, Phys. Rev. A 45, 8185 (1992).

[13] D. L. Andrews, Structured Light and Its Applications: An Introduction to Phase-Structured Beams and Nanoscale Optical Forces (Academic Press, Burlington, 2011).

[14] A. M. Yao and M. J. Padgett, Orbital angular momentum: Origins, behavior and applications, Adv. Opt. Photonics 3, 161 (2011).

[15] D. L. Andrews and M. Babiker, The Angular Momentum of Light (Cambridge University Press, Cambridge, 2012).

[16] H. Rubinsztein-Dunlop et al., Roadmap on structured light, J. Opt. 19, 013001 (2016).

[17] S. M. Barnett, M. Babiker, and M. J. Padgett, Optical Orbital Angular Momentum (The Royal Society, London, 2017).

[18] Y. Yue, H. Huang, Y. Ren, Z. Pan, and A. E. Willner, Special issue on novel insights into orbital angular momentum beams: From fundamentals, devices to applications, Appl. Sci. 9, 2600 (2019).

[19] D. L. Andrews, L. D. Romero, and M. Babiker, On optical vortex interactions with chiral matter, Opt. Commun. 237, 133 (2004).

[20] L. Allen, Orbital angular momentum: A personal memoir, Philos. Trans. R. Soc., A 375, 20160280 (2017).

[21] F. Araoka, T. Verbiest, K. Clays, and A. Persoons, Interactions of twisted light with chiral molecules: An experimental investigation, Phys. Rev. A 71, 055401 (2005).

[22] W. Löffler, D. J. Broer, and J. P. Woerdman, Circular dichroism of cholesteric polymers and the orbital angular momentum of light, Phys. Rev. A 83, 065801 (2011).

[23] W. Löffler, M. P. Van Exter, G. Nienhuis, D. J. Broer, and J. P. Woerdman, Search for Hermite-Gauss mode 
rotation in cholesteric liquid crystals, Opt. Express 19, 12978 (2011).

[24] V. E. Lembessis and M. Babiker, Enhanced Quadrupole Effects for Atoms in Optical Vortices, Phys. Rev. Lett. 110, 083002 (2013).

[25] M. G. Mandujano and J. A. Maytorena, Quadrupolar secondharmonic generation by helical beams and vectorial vortices with radial or azimuthal polarization, Phys. Rev. A 88, 023811 (2013).

[26] K. A. Forbes and D. L. Andrews, Optical orbital angular momentum: Twisted light and chirality, Opt. Lett. 43, 435 (2018).

[27] K. A. Forbes and D. L. Andrews, Spin-orbit interactions and chiroptical effects engaging orbital angular momentum of twisted light in chiral and achiral media, Phys. Rev. A 99, 023837 (2019).

[28] K. A. Forbes, Raman Optical Activity Using Twisted Photons, Phys. Rev. Lett. 122, 103201 (2019).

[29] K. Y. Bliokh, F. J. Rodríguez-Fortuño, F. Nori, and A. V. Zayats, Spin-orbit interactions of light, Nat. Photonics 9, 796 (2015).

[30] X. Zambrana-Puyalto, X. Vidal, and G. Molina-Terriza, Angular momentum-induced circular dichroism in non-chiral nanostructures, Nat. Commun. 5, 4922 (2014).

[31] A. Afanasev, C. E. Carlson, and M. Solyanik, Circular dichroism of twisted photons in non-chiral atomic matter, J. Opt. 19, 105401 (2017).

[32] J. Ni, Z. Wang, Z. Lao, Y. Hu, K. Huang, S. Ji, J. Li, Z. Huang, D. $\mathrm{Wu}$, and C. Qiu, Ultra-sensitive experimental detection of chiral meso-structures by orbital angular momentum of light, arXiv: 1809.02754

[33] C. T. Samlan, R. R. Suna, D. N. Naik, and N. K. Viswanathan, Spin-orbit beams for optical chirality measurement, Appl. Phys. Lett. 112, 031101 (2018).

[34] A. A. Sirenko, P. Marsik, C. Bernhard, T. N. Stanislavchuk, V. Kiryukhin, and S.-W. Cheong, Terahertz Vortex Beam as a Spectroscopic Probe of Magnetic Excitations, Phys. Rev. Lett. 122, 237401 (2019).

[35] T. Wu, R. Wang, and X. Zhang, Plasmon-induced strong interaction between chiral molecules and orbital angular momentum of light, Sci. Rep. 5, 18003 (2015).

[36] W. Brullot, M. K. Vanbel, T. Swusten, and T. Verbiest, Resolving enantiomers using the optical angular momentum of twisted light, Sci. Adv. 2, e1501349 (2016).

[37] S. Wang, Z.-L. Deng, Y. Cao, D. Hu, Y. Xu, B. Cai, L. Jin, Y. Bao, X. Wang, and X. Li, Angular momentum-dependent transmission of circularly polarized vortex beams through a plasmonic coaxial nanoring, IEEE Photonics J. 10, 5700109 (2018).

[38] H. Ren and M. Gu, Angular momentum-reversible near-unity bisignate circular dichroism, Laser Photonics Rev. 12, 1700255 (2018).

[39] I. V. Reddy, A. Baev, E. P. Furlani, P. N. Prasad, and J. W. Haus, Interaction of structured light with a chiral plasmonic metasurface: Giant enhancement of chiro-optic response, ACS Photonics 5, 734 (2018).

[40] R. M. Kerber, J. M. Fitzgerald, S. S. Oh, D. E. Reiter, and O. Hess, Orbital angular momentum dichroism in nanoantennas, Commun. Phys. 1, 87 (2018).

[41] L. Ye, J. R. Rouxel, S. Asban, B. Rösner, and S. Mukamel, Probing molecular chirality by orbital angular momentum carrying X-ray pulses, J. Chem. Theory Comput. 15, 4180 (2019).
[42] L. D. Barron and A. D. Buckingham, Rayleigh and Raman scattering from optically active molecules, Mol. Phys. 20, 111 (1971).

[43] L. D. Barron, S. T. Mutter, and E. W. Blanch, Raman optical activity, in Chiral Analysis: Advances in Spectroscopy, Chromatography and Emerging Methods, 2nd ed. (Elsevier, New York, 2018), pp. 249-291.

[44] L. D. Barron, L. Hecht, E. W. Blanch, and A. F. Bell, Solution structure and dynamics of biomolecules from Raman optical activity, Prog. Biophys. Mol. Biol. 73, 1 (2000).

[45] L. D. Barron, E. W. Blanch, and L. Hecht, Unfolded proteins studied by Raman optical activity, Adv. Protein Chem. 62, 51 (2002).

[46] L. D. Barron, E. W. Blanch, I. H. McColl, C. D. Syme, L. Hecht, and K. Nielsen, Structure and behaviour of proteins, nucleic acids and viruses from vibrational Raman optical activity, J. Spectrosc. 17, 101 (2003).

[47] L. D. Barron, L. Hecht, I. H. McColl, and E. W. Blanch, Raman optical activity comes of age, Mol. Phys. 102, 731 (2004).

[48] S. Yamamoto, Conformational analyses of peptides and proteins by vibrational Raman optical activity, Anal. Bioanal. Chem. 403, 2203 (2012).

[49] V. Parchaňský, J. Kapitan, and P. Bouř, Inspecting chiral molecules by Raman optical activity spectroscopy, RSC Adv. 4, 57125 (2014).

[50] S. Luber, Exploring Raman optical activity for transition metals: From coordination compounds to solids, Biomed. Spectrosc. Imaging 4, 255 (2015).

[51] S. T. Mutter, F. Zielinski, J. R. Cheeseman, C. Johannessen, P. L. Popelier, and E. W. Blanch, Conformational dynamics of carbohydrates: Raman optical activity of D-glucuronic acid and $\mathrm{N}$-acetyl-D-glucosamine using a combined molecular dynamics and quantum chemical approach, Phys. Chem. Chem. Phys. 17, 6016 (2015).

[52] L. D. Barron, The development of biomolecular Raman optical activity spectroscopy, Biomed. Spectrosc. Imaging 4, 223 (2015).

[53] P. L. Polavarapu, Chiral Analysis: Advances in Spectroscopy, Chromatography and Emerging Methods, 2nd ed. (Elsevier Science, New York, 2018).

[54] S. Yamamoto, J. Kaminský, and P. Bouř, Structure and vibrational motion of insulin from Raman optical activity spectra, Anal. Chem. 84, 2440 (2012).

[55] S. Abdali and E. W. Blanch, Surface enhanced Raman optical activity (SEROA), Chem. Soc. Rev. 37, 980 (2008).

[56] R. Acevedo, R. Lombardini, N. J. Halas, and B. R. Johnson, Plasmonic enhancement of Raman optical activity in molecules near metal nanoshells, J. Phys. Chem. A 113, 13173 (2009).

[57] M. Schäferling, D. Dregely, M. Hentschel, and H. Giessen, Tailoring Enhanced Optical Chirality: Design Principles for Chiral Plasmonic Nanostructures, Phys. Rev. X 2, 031010 (2012).

[58] H. Rhee, J. S. Choi, D. J. Starling, J. C. Howell, and M. Cho, Amplifications in chiroptical spectroscopy, optical enantioselectivity, and weak value measurement, Chem. Sci. 4, 4107 (2013).

[59] R.-Y. Wang, P. Wang, Y. Liu, W. Zhao, D. Zhai, X. Hong, Y. Ji, X. Wu, F. Wang, and D. Zhang, Experimental observation of giant chiroptical amplification of small chiral molecules by gold nanosphere clusters, J. Phys. Chem. C 118, 9690 (2014). 
[60] S. S. Oh and O. Hess, Chiral metamaterials: Enhancement and control of optical activity and circular dichroism, Nano Converg. 2, 24 (2015).

[61] J. R. Rouxel, Y. Zhang, and S. Mukamel, X-ray Raman optical activity of chiral molecules, Chem. Sci. 10, 898 (2019).

[62] D. L. Andrews, G. A. Jones, A. Salam, and R. G. Woolley, Perspective: Quantum Hamiltonians for optical interactions, J. Chem. Phys. 148, 040901 (2018).

[63] D. L. Andrews and T. Thirunamachandran, On threedimensional rotational averages, J. Chem. Phys. 67, 5026 (1977).

[64] D. L. Andrews, Rayleigh and Raman optical activity: An analysis of the dependence on scattering angle, J. Chem. Phys. 72, 4141 (1980).

[65] J. Li, J. J. Tu, and J. L. Birman, Raman scattering using vortex light, J. Phys. Chem. Solids 77, 117 (2015).

[66] G. Campbell, B. Hage, B. Buchler, and P. K. Lam, Generation of high-order optical vortices using directly machined spiral phase mirrors, Appl. Opt. 51, 873 (2012).

[67] L. Hecht and L. D. Barron, An analysis of modulation experiments for Raman optical activity, Appl. Spectrosc. 44, 483 (1990).

[68] J.-F. Bisson, K. Miyamoto, and T. Omatsu, Power-scalable and high-speed orbital angular momentum modulator, Jpn. J. Appl. Phys. 58, 032009 (2019).

[69] B. Paroli, M. Siano, and M. A. C. Potenza, The local intrinsic curvature of wavefronts allows to detect optical vortices, Opt. Express 27, 17550 (2019).

[70] N. B. Simpson, K. Dholakia, L. Allen, and M. J. Padgett, Mechanical equivalence of spin and orbital angular momentum of light: An optical spanner, Opt. Lett. 22, 52 (1997).

[71] A. T. O’Neil, I. MacVicar, L. Allen, and M. J. Padgett, Intrinsic and Extrinsic Nature of the Orbital Angular Momentum of a Light Beam, Phys. Rev. Lett. 88, 053601 (2002).

[72] V. Garcés-Chávez, D. McGloin, M. J. Padgett, W. Dultz, H. Schmitzer, and K. Dholakia, Observation of the Transfer of the Local Angular Momentum Density of a Multiringed Light Beam to an Optically Trapped Particle, Phys. Rev. Lett. 91, 093602 (2003).

[73] O. V. Angelsky, A. Y. Bekshaev, P. P. Maksimyak, A. P. Maksimyak, S. G. Hanson, and C. Y. Zenkova, Orbital rotation without orbital angular momentum: Mechanical action of the spin part of the internal energy flow in light beams, Opt. Express 20, 3563 (2012).

[74] J. Courtial, R. Zambrini, M. R. Dennis, and M. Vasnetsov, Angular momentum of optical vortex arrays, Opt. Express 14, 938 (2006).
[75] L. Allen and M. Padgett, Equivalent geometric transformations for spin and orbital angular momentum of light, J. Mod. Opt. 54, 487 (2007).

[76] A. S. Ostrovsky, C. Rickenstorff-Parrao, and V. Arrizón, Generation of the perfect optical vortex using a liquid-crystal spatial light modulator, Opt. Lett. 38, 534 (2013).

[77] M. Chen, M. Mazilu, Y. Arita, E. M. Wright, and K. Dholakia, Dynamics of microparticles trapped in a perfect vortex beam, Opt. Lett. 38, 4919 (2013).

[78] J. García-García, C. Rickenstorff-Parrao, R. Ramos-García, V. Arrizón, and A. S. Ostrovsky, Simple technique for generating the perfect optical vortex, Opt. Lett. 39, 5305 (2014).

[79] P. Vaity and L. Rusch, Perfect vortex beam: Fourier transformation of a Bessel beam, Opt. Lett. 40, 597 (2015).

[80] P. Figliozzi, N. Sule, Z. Yan, Y. Bao, S. Burov, S. K. Gray, S. A. Rice, S. Vaikuntanathan, and N. F. Scherer, Driven optical matter: Dynamics of electrodynamically coupled nanoparticles in an optical ring vortex, Phys. Rev. E 95, 022604 (2017).

[81] Y. Chen, Z.-X. Fang, Y.-X. Ren, L. Gong, and R.-D. Lu, Generation and characterization of a perfect vortex beam with a large topological charge through a digital micromirror device, Appl. Opt. 54, 8030 (2015).

[82] C. T. Schmiegelow, J. Schulz, H. Kaufmann, T. Ruster, U. G. Poschinger, and F. Schmidt-Kaler, Transfer of optical orbital angular momentum to a bound electron, Nat. Commun. 7, 12998 (2016).

[83] M. Babiker, D. L. Andrews, and V. E. Lembessis, Atoms in complex twisted light, J. Opt. 21, 013001 (2019).

[84] E. Bendau, L. Zhang, R. Gozali, S. Ashrafi, and R. R. Alfano, Vortex beams and optical activity of sucrose, Proc. SPIE 10120, 1012004 (2017).

[85] G. Milione, J. Secor, G. Michel, S. Evans, and R. R. Alfano, Raman optical activity by light with spin and orbital angular momentum, Proc. SPIE 7950, 79500H (2011).

[86] S. Mamani, L. Shi, T. Ahmed, R. Karnik, A. RodríguezContreras, D. Nolan, and R. Alfano, Transmission of classically entangled beams through mouse brain tissue, J. Biophotonics 11, e201800096 (2018).

[87] S. T. Mutter, F. Zielinski, P. L. Popelier, and E. W. Blanch, Calculation of Raman optical activity spectra for vibrational analysis, Analyst 140, 2944 (2015).

[88] M. Srebro-Hooper and J. Autschbach, Calculating natural optical activity of molecules from first principles, Annu. Rev. Phys. Chem. 68, 399 (2017)

[89] H. Li and L. A. Nafie, Simultaneous acquisition of all four forms of circular polarization Raman optical activity: Results for $\alpha$-pinene and lysozyme, J. Raman Spectrosc. 43, 89 (2012). 